\title{
Assessment of Concrete Compressive Strength by Ultrasonic Non- Destructive Test
}

\author{
AbdulMuttalib I. Said ${ }^{1, a^{*}}$ and Baqer Abdul Hussein Ali ${ }^{1, b}$ \\ ${ }^{1}$ Department of Civil Engineering, University of Baghdad, Baghdad, Iraq. \\ aDr.AbdulMuttalib.I.Said@coeng.uobaghdad.edu.iq, bbaqer1968@gmail.com
}

\begin{abstract}
This paper has carried out an experimental program to establish a relatively accurate relation between the ultrasonic pulse velocity (UPV) and the concrete compressive strength. The program involved testing concrete cubes of $(100) \mathrm{mm}$ and prisms of $(100 \times 100 \times 300)$ cast with specified test variables. The samples are tested by using ultrasonic test equipment with two methods, direct ultrasonic pulse (DUPV) and surface (indirect) ultrasonic pulse (SUPV) for each sample. The obtained results were used as input data in the statistical program (SPSS) to predict the best equation representing the relation between the compressive strength and the ultrasonic pulse velocity. In this research 383 specimens were tested, and an exponential equation is proposed for this purpose. The statistical program has been used to prove which type of UPV is more suitable, the (SUPV) test or the (DUPV) test, to represent the relation between the ultrasonic pulse velocity and the concrete compressive strength. In this paper, the effect of salt content on the connection between the ultrasonic pulse velocity and the concrete compressive strength has also been studied.
\end{abstract}

Keywords: Assessment; evaluation; concrete structures; compressive strength; non-destructive test; ultrasonic pulse velocity method.

\section{Introduction}

The (UPV) method estimates the compressive strength of concrete by measuring the (UPV) from the pulse passing the time between the transmitter and receiver at certain distances in a concrete structure. The ultrasonic pulse velocity can evaluate concrete quality factors such as the compressive strength by obtaining a correlation between the (UPV) and the compressive strength. This study aims to correlate the compressive strength of the tested cubes and the results of the non-destructive tests (UPV) for the prism cast from the same concrete mix by using statistical methods.

Several studies have been made to develop the relationship between the ultrasonic pulse velocity and the compressive strength;

- Jones R. Equation in (1962) [1]. $C=2.8 e^{0.53 D}$

- Elvery and Ibrahim Equation in (1976) [2]. $C=0.0012 e^{2.27 D} \ldots \pm 6.4$

- Raouf, Z and Ali Z.M. Equation in (1983) [3]. $C=2.016 e^{0.61 D}$

- Popovics et al. Equation in (1990) [4]. $C=0.0028 e^{0.0021 D}$

- Nash't et al. equation in (2005) [5]. $C=1.19 e^{0.715 D}$

- Jassim, A.K et al. equation in (2013) $[6,7] C=0.173 e^{1.157 D}, C=0.46 e^{1.16 S}$

Where $\mathrm{C}$ : compressive strength in $\mathrm{MPa}$ and $\mathrm{D}$ : direct ultrasonic velocity in $\mathrm{km} / \mathrm{sec}$

\section{Experimental Results}

The study covers 383 test results on 172 prisms and nearly 900 concrete cubes of $100 \mathrm{~mm}$ [8]. These cubes were a product from the mixtures designed for this research using ordinary Portland cement compatible with the Iraqi standard (No.5) and sulfate resisting Portland cement with different curing conditions. The mixing properties and the experimental results are shown in Table 1. For this 
research, different graded and maximum size coarse aggregate are prepared to satisfy the grading requirements. The coarse aggregate grading and characteristics are given in Table 2.

\section{Discussion of Experimental Work Results}

The ultrasonic pulse velocity (UPV) had been measured in two ways, the first one was (SUPV) and the second was (DUPV) in order to find the best relation which can be correlated to the compressive strength. A comparison had been made to find the best form of the (UPV) type: surface ultrasonic pulse velocity (SUPV) or direct ultrasonic pulse velocity (DUPV) to represent the relation between UPV and the compressive strength. To investigate the SUPV and DUPV, data have been drawn with the compressive strength for all the samples subjected to normal curing. Two exponential curves were found, as shown in Figures 1 and 2 . The correlation factor and $\mathrm{R}^{2}$ were found for the two curves, as shown in Table 3.

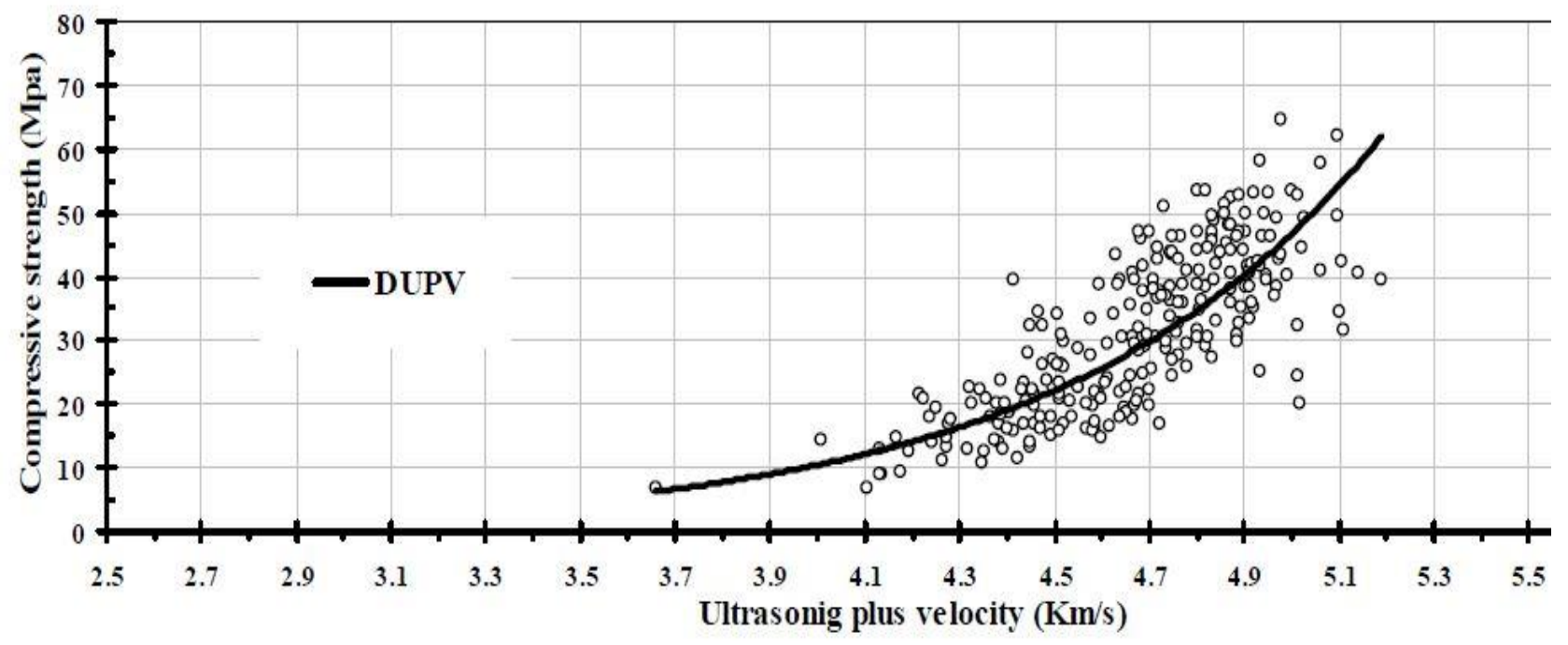

Figure 1. Relation between (DUPV) with the compressive strength for concrete samples subjected to normal curing.

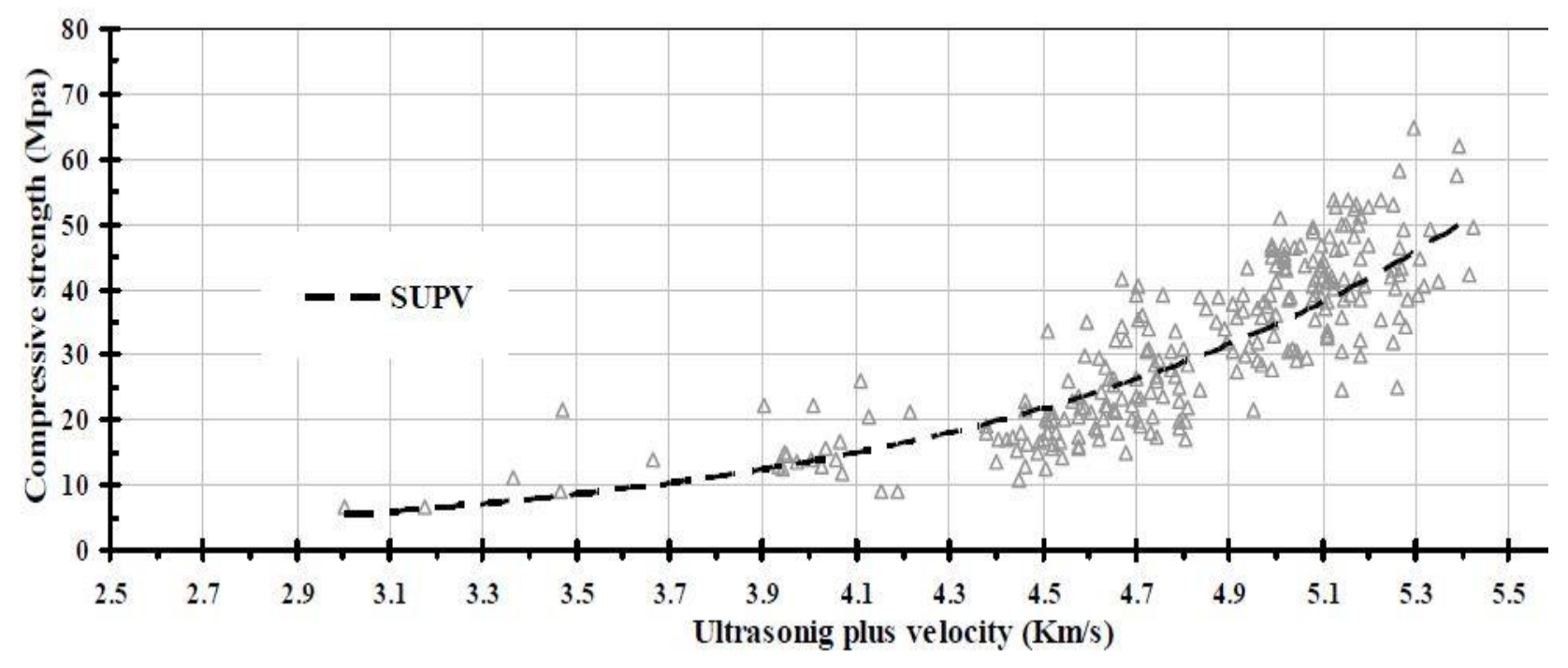

Figure 2. Relation between (SUPV) with the compressive strength for concrete samples subjected to normal curing. 
Table 1. Experimental results of cubes and prism (normally curing).

\begin{tabular}{|c|c|c|c|c|c|c|c|c|c|c|c|}
\hline $\begin{array}{c}\text { Sample } \\
\text { no. }\end{array}$ & $\begin{array}{c}\text { SLUMP } \\
(\mathrm{mm})\end{array}$ & $\begin{array}{c}\text { SLUMP } \\
\text { range } \\
(\mathrm{mm})\end{array}$ & $\begin{array}{c}\mathrm{SO}_{3} \% \\
\text { in fine } \\
\text { agregate }\end{array}$ & $\mathbf{W} / \mathbf{C}$ & $\begin{array}{c}\text { Coarse } \\
\text { Aggregate }\end{array}$ & $\begin{array}{c}\text { Mix } \\
\text { proportions }\end{array}$ & $\begin{array}{l}\text { Age } \\
\text { (day) }\end{array}$ & $\begin{array}{c}\text { Comp. } \\
\text { str. } \\
\text { (Mpa) }\end{array}$ & $\begin{array}{c}\text { Ult. } \\
\text { V(km/s) } \\
\text { direct }\end{array}$ & $\begin{array}{c}\text { Ult. } \\
\text { V(km/s)s } \\
\text { urface }\end{array}$ & $\begin{array}{c}\text { Density } \\
(\mathrm{gm} / \mathrm{cm} 3)\end{array}$ \\
\hline 1 & 90 & \begin{tabular}{|c|}
$(60-180)$ \\
\end{tabular} & 0.34 & 0.6 & Type 1 & $1: 2.09: 2.66$ & 7 & 7.05 & 4.26 & 3.36 & 2.42 \\
\hline 2 & 90 & \begin{tabular}{|c|}
$(60-180)$ \\
\end{tabular} & 0.34 & 0.6 & Type 1 & $1: 2.09: 2.66$ & 14 & \begin{tabular}{|l|}
13.35 \\
\end{tabular} & 4.58 & 3.98 & 2.42 \\
\hline 3 & 90 & \begin{tabular}{|c|}
$(60-180)$ \\
\end{tabular} & 0.34 & 0.6 & Type 1 & $1: 2.09: 2.66$ & 21 & 23.00 & 4.54 & 4.51 & 2.39 \\
\hline 4 & 90 & \begin{tabular}{|c|}
$(60-180)$ \\
\end{tabular} & 0.34 & 0.6 & Type 1 & $1: 2.09: 2.66$ & 28 & 27.25 & 4.60 & 4.68 & 2.41 \\
\hline 5 & 90 & \begin{tabular}{|c|}
$(60-180)$ \\
\end{tabular} & 0.34 & 0.6 & Type 1 & $1: 2.09: 2.66$ & 60 & 30.78 & 4.65 & 4.80 & 2.43 \\
\hline 6 & 90 & \begin{tabular}{|c|}
$(60-180)$ \\
\end{tabular} & 0.34 & 0.6 & Type 1 & 1:2.09:2.66 & 90 & 30.77 & 4.69 & 4.79 & 2.39 \\
\hline 7 & 90 & \begin{tabular}{|c|}
$(60-180)$ \\
\end{tabular} & 0.34 & 0.6 & Type 1 & $1: 2.09: 2.66$ & 120 & 31.13 & 4.70 & 4.81 & 2.40 \\
\hline 8 & 68 & \begin{tabular}{|c|}
$(60-180)$ \\
\end{tabular} & 0.34 & 0.4 & Type 2 & 1:1.13:1.7 & 14 & \begin{tabular}{|l|}
31.92 \\
\end{tabular} & 4.68 & 4.90 & 2.33 \\
\hline 9 & 68 & \begin{tabular}{|c|}
$(60-180)$ \\
\end{tabular} & 0.34 & 0.4 & Type 2 & 1:1.13:1.7 & 21 & 43.75 & 4.74 & 5.00 & 2.35 \\
\hline 10 & 68 & \begin{tabular}{|c|}
$(60-180)$ \\
\end{tabular} & 0.34 & 0.4 & Type 2 & 1:1.13:1.7 & 28 & 43.30 & 4.75 & 5.02 & 2.35 \\
\hline 11 & 68 & \begin{tabular}{|l|}
$(60-180)$ \\
\end{tabular} & 0.34 & 0.4 & Type 2 & 1:1.13:1.7 & 60 & 48.66 & 4.84 & 5.08 & 2.36 \\
\hline 12 & 68 & $(60-180)$ & 0.34 & 0.4 & Type 2 & 1:1.13:1.7 & 90 & 46.43 & 4.83 & 5.04 & 2.35 \\
\hline 13 & 56 & \begin{tabular}{|c|}
$(30-60)$ \\
\end{tabular} & 0.34 & 0.65 & Type 2 & $1: 2.31: 3.47$ & 14 & 18.12 & 4.34 & 4.57 & 2.33 \\
\hline 14 & 56 & $(30-60)$ & 0.34 & 0.65 & Type 2 & $1: 2.31: 3.47$ & 21 & 20.77 & 4.18 & 4.61 & 2.33 \\
\hline 15 & 56 & $(30-60)$ & 0.34 & 0.65 & Type 2 & $1: 2.31: 3.47$ & 28 & 23.42 & 4.44 & 4.65 & 2.33 \\
\hline 16 & 56 & $(30-60)$ & 0.34 & 0.65 & Type 2 & $1: 2.31: 3.47$ & 60 & 28.29 & 4.50 & 4.69 & 2.32 \\
\hline 17 & 56 & $(30-60)$ & 0.34 & 0.65 & Type 2 & $1: 2.31: 3.47$ & 90 & 27.40 & 4.53 & 4.73 & 2.33 \\
\hline 18 & 10 & $(0-10)$ & 0.34 & 0.4 & Type 5 & $1: 1.36: 3.03$ & 7 & 37.72 & 4.72 & 4.91 & 2.47 \\
\hline 19 & 10 & $(0-10)$ & 0.34 & 0.4 & Type 5 & $1: 1.36: 3.03$ & 14 & 48.21 & 4.87 & 5.17 & 2.51 \\
\hline 20 & 10 & $(0-10)$ & 0.34 & 0.4 & Type 5 & $1: 1.36: 3.03$ & 21 & \begin{tabular}{|l|}
46.88 \\
\end{tabular} & 4.90 & 5.20 & 2.52 \\
\hline 21 & 10 & $(0-10)$ & 0.34 & 0.4 & Type 5 & $1: 1.36: 3.03$ & 28 & $\mathbf{5 8 . 0 4}$ & 4.93 & 5.27 & 2.52 \\
\hline 22 & 10 & $(0-10)$ & 0.34 & 0.4 & Type 5 & $1: 1.36: 3.03$ & 60 & 64.73 & 4.98 & 5.30 & 2.50 \\
\hline 23 & 10 & $(0-10)$ & 0.34 & 0.4 & Type 5 & $1: 1.36: 3.03$ & 90 & 49.11 & 5.03 & 5.33 & 2.50 \\
\hline 24 & 27 & $(10-30)$ & 0.34 & 0.4 & Type 5 & $1: 1.26: 2.45$ & 7 & 39.29 & 4.42 & 4.99 & 2.44 \\
\hline 25 & 27 & $(10-30)$ & 0.34 & 0.4 & Type 5 & $1: 1.26: 2.45$ & 14 & 44.20 & 4.80 & 5.02 & 2.41 \\
\hline 26 & 27 & $(10-30)$ & 0.34 & 0.4 & Type 5 & $1: 1.26: 2.45$ & 21 & \begin{tabular}{|l|}
46.88 \\
\end{tabular} & 4.83 & 5.06 & 2.43 \\
\hline 27 & 27 & $(10-30)$ & 0.34 & 0.4 & Type 5 & 1:1.26:2.45 & 28 & 48.21 & 4.87 & 5.12 & 2.44 \\
\hline 28 & 27 & $(\mathbf{1 0 - 3 0})$ & 0.34 & 0.4 & Type 5 & $1: 1.26: 2.45$ & 60 & 46.43 & 4.94 & 5.14 & 2.43 \\
\hline 29 & 27 & \begin{tabular}{|l|}
$(10-30)$ \\
\end{tabular} & 0.34 & 0.4 & Type 5 & $1: 1.26: 2.45$ & 90 & 50.00 & 4.94 & 5.14 & 2.42 \\
\hline 30 & 27 & \begin{tabular}{|l|}
$(10-30)$ \\
\end{tabular} & 0.34 & 0.4 & Type 5 & $1: 1.26: 2.45$ & 100 & 34.82 & & 4.60 & \\
\hline 31 & 73 & \begin{tabular}{|c|}
$(60-180)$ \\
\end{tabular} & 0.34 & 0.5 & Type 4 & 1:1.91:2.25 & 150 & 43.75 & $4 \overline{85}$ & 5.05 & 2.38 \\
\hline 32 & 73 & \begin{tabular}{|c|}
$(60-180)$ \\
\end{tabular} & 0.34 & 0.5 & Type 4 & $1: 1.91: 2.25$ & 7 & 28.06 & 4.63 & 4.76 & 2.36 \\
\hline 33 & 73 & \begin{tabular}{|l|}
$(60-180)$ \\
\end{tabular} & 0.34 & 0.5 & Type 4 & $1: 1.91: 2.25$ & 14 & 29.61 & 4.69 & 4.88 & 2.33 \\
\hline 34 & 73 & $(60-180)$ & 0.34 & 0.5 & Type 4 & 1:1.91:2.25 & 21 & 31.38 & 4.76 & 4.91 & 2.38 \\
\hline 35 & 73 & \begin{tabular}{|c|}
$(60-180)$ \\
\end{tabular} & 0.34 & 0.5 & Type 4 & $1: 1.91: 2.25$ & 28 & 38.45 & 4.76 & 4.98 & 2.39 \\
\hline 36 & 73 & \begin{tabular}{|l|}
$(60-180)$ \\
\end{tabular} & 0.34 & 0.5 & Type 4 & $1: 1.91: 2.25$ & 60 & 43.31 & 4.80 & 5.01 & 2.38 \\
\hline 37 & 73 & \begin{tabular}{|c|}
$(60-180)$ \\
\end{tabular} & 0.34 & 0.5 & Type 4 & $1: 1.91: 2.25$ & 90 & 40.22 & 4.82 & 5.03 & 2.37 \\
\hline 38 & 73 & \begin{tabular}{|c|}
$(60-180)$ \\
\end{tabular} & 0.34 & 0.5 & Type 4 & $1: 1.91: 2.25$ & 120 & 43.75 & 4.83 & 5.03 & 2.37 \\
\hline 39 & 59 & \begin{tabular}{|c|}
$(30-60)$ \\
\end{tabular} & 0.34 & 0.4 & Type 2 & 1:1.17:1.93 & 7 & 42.86 & 4.72 & 5.02 & 2.46 \\
\hline 40 & 59 & $(30-60)$ & 0.34 & 0.4 & Type 2 & $1: 1.17: 1.93$ & 14 & 43.75 & 4.75 & 5.10 & 2.44 \\
\hline 41 & 59 & $(30-60)$ & 0.34 & 0.4 & Type 2 & 1:1.17:1.93 & 21 & $\mathbf{5 3 . 5 7}$ & 4.80 & 5.13 & 2.44 \\
\hline 42 & 59 & $(30-60)$ & 0.34 & 0.4 & Type 2 & 1:1.17:1.93 & 28 & $\mathbf{5 3 . 5 7}$ & 4.82 & 5.16 & 2.46 \\
\hline 43 & 59 & $(30-60)$ & 0.34 & 0.4 & Type 2 & $1: 1.17: 1.93$ & 60 & 52.23 & 4.87 & 5.17 & 2.44 \\
\hline 44 & 59 & $(30-60)$ & 0.34 & 0.4 & Type 2 & 1:1.17:1.93 & 90 & 50.00 & 4.90 & 5.15 & 2.44 \\
\hline 45 & 95 & \begin{tabular}{|c|}
$(60-180)$ \\
\end{tabular} & 0.34 & 0.8 & Type 4 & $1: 3.35: 4.27$ & 7 & 13.07 & 4.19 & 3.94 & 2.39 \\
\hline 46 & 95 & \begin{tabular}{|c|}
$(60-180)$ \\
\end{tabular} & 0.34 & 0.8 & Type 4 & $1: 3.35: 4.27$ & 90 & 28.39 & 4.69 & 4.70 & 2.37 \\
\hline 47 & 95 & \begin{tabular}{|c|}
$(60-180)$ \\
\end{tabular} & 0.34 & 0.8 & Type 4 & $1: 3.35: 4.27$ & 14 & 22.88 & 4.54 & 4.49 & 2.39 \\
\hline 48 & 95 & \begin{tabular}{|l|}
$(60-180)$ \\
\end{tabular} & 0.34 & 0.8 & Type 4 & $1: 3.35: 4.27$ & 28 & 26.47 & 4.61 & 4.63 & 2.37 \\
\hline 49 & 95 & \begin{tabular}{|c|}
$(60-180)$ \\
\end{tabular} & 0.34 & 0.8 & Type 4 & 1:3.35:4.27 & 60 & 27.67 & 4.68 & 4.67 & 2.37 \\
\hline 50 & 78 & \begin{tabular}{|c|}
$(60-180)$ \\
\end{tabular} & 0.34 & 0.45 & Type 1 & 1:1.47:1.86 & 7 & 34.38 & 4.47 & 4.67 & 2.37 \\
\hline 51 & 78 & \begin{tabular}{|l|}
$(60-180)$ \\
\end{tabular} & 0.34 & 0.45 & Type 1 & $1: 1.47: 1.86$ & 14 & 33.48 & 4.58 & 4.79 & 2.37 \\
\hline 52 & 78 & \begin{tabular}{|l|}
$(60-180)$ \\
\end{tabular} & 0.34 & 0.45 & Type 1 & $1: 1.47: 1.86$ & 21 & 33.93 & 4.63 & 4.89 & 2.38 \\
\hline 53 & 78 & \begin{tabular}{|c|}
$(60-180)$ \\
\end{tabular} & 0.34 & 0.45 & Type 1 & 1:1.47:1.86 & 28 & 39.29 & 4.64 & 4.93 & 2.37 \\
\hline 54 & 78 & \begin{tabular}{|c|}
$(60-180)$ \\
\end{tabular} & 0.34 & 0.45 & Type 1 & 1:1.47:1.86 & 60 & 44.64 & 4.72 & 5.02 & 2.36 \\
\hline 55 & 78 & \begin{tabular}{|l|}
$(60-180)$ \\
\end{tabular} & 0.34 & 0.45 & Type 1 & 7:1.86 & 90 & 46.43 & 4.77 & 4.99 & 2.36 \\
\hline 56 & 78 & $(60-180)$ & 0.34 & 0.45 & Type 1 & 1:1.47:1.86 & 120 & 46.43 & 4.75 & 4.99 & 2.36 \\
\hline 57 & 55 & $(30-60)$ & 0.34 & 0.45 & Type 3 & 1:1.4:2.29 & 7 & 29.91 & 4.52 & 4.59 & 2.34 \\
\hline 58 & 55 & (30) & 0.34 & 0.45 & Type 3 & 1:1 & 14 & 30.58 & 4.67 & 4.77 & 2.34 \\
\hline 59 & 55 & $(30-60)$ & 0.34 & 0.45 & Type 3 & 1:1.4:2.29 & 21 & 34.82 & 4.69 & 4.87 & 2.33 \\
\hline 60 & 55 & $(30-60)$ & 0.34 & 0.45 & Type 3 & 1:1.4:2.29 & 28 & 36.61 & 4.72 & 4.93 & 2.35 \\
\hline 61 & 55 & $(30-60)$ & 0.34 & 0.45 & Type 3 & 1:1.4:2.29 & 60 & 46.88 & 4.80 & 5.02 & 2.33 \\
\hline 62 & 55 & & 0.34 & 0.45 & Type 3 & & 90 & 45.54 & 4.83 & 5.02 & 2.35 \\
\hline 63 & 55 & $(30-60)$ & 0.34 & 0.45 & Type 3 & 1:1.4:2.29 & 120 & 45.09 & 4.86 & 4.99 & 2.35 \\
\hline 64 & 29 & $(10-30)$ & 0.34 & 0.45 & Type 2 & 1:1.51:2.79 & 7 & 26.34 & 4.50 & 4.70 & 2.41 \\
\hline 65 & 29 & $(10-30)$ & 0.34 & 0.45 & Type 2 & $1: 1.51: 2.79$ & 14 & 37.50 & 4.69 & 4.98 & 2.42 \\
\hline 66 & 29 & $(10-30)$ & 0.34 & 0.45 & Type 2 & $1: 1.51: 2.79$ & 21 & 38.39 & 4.74 & 5.03 & 2.41 \\
\hline 67 & 29 & $(10-30)$ & 0.34 & 0.45 & Type 2 & 1:1.51:2.79 & 28 & 42.86 & 4.76 & 5.10 & 2.41 \\
\hline 68 & 29 & \begin{tabular}{|l|}
$(10-30)$ \\
\end{tabular} & 0.34 & 0.45 & Type 2 & $1: 1.51: 2.79$ & 60 & 44.64 & 4.82 & 5.18 & 2.42 \\
\hline 69 & 29 & $(10-30$ & 0.34 & 0.45 & Type 2 & $1: 1.51: 2.79$ & 90 & \begin{tabular}{|l|}
51.34 \\
\end{tabular} & 4.86 & 5.18 & 2.41 \\
\hline 70 & 29 & \begin{tabular}{|l|}
$(10-30)$ \\
\end{tabular} & 0.34 & 0.45 & Type 2 & $1: 1.51: 2.79$ & 120 & 50.00 & 4.86 & 5.18 & 2.43 \\
\hline 71 & 56 & $(30-60)$ & 0.34 & 0.4 & Type 1 & 1:1.17:1.93 & 90 & 49.50 & 4.85 & 5.05 & 2.44 \\
\hline 72 & 56 & $(30-60)$ & 0.34 & 0.4 & Type 1 & $1: 1.17: 1.93$ & 7 & \begin{tabular}{|l|}
42.43 \\
\end{tabular} & 4.67 & 4.92 & 2.46 \\
\hline 73 & 56 & $(30-60)$ & 0.34 & 0.4 & Type 1 & $1: 1.17: 1.93$ & 14 & 43.31 & 4.70 & 4.99 & 2.44 \\
\hline 74 & 56 & \begin{tabular}{|l|}
$(30-60)$ \\
\end{tabular} & 0.34 & 0.4 & Type 1 & 1:1.17:1.93 & 21 & \begin{tabular}{|l|}
53.04 \\
\end{tabular} & 4.76 & 5.02 & 2.44 \\
\hline 75 & 56 & $(30-60)$ & 0.34 & 0.4 & Type 1 & 1:1.17:1.93 & 28 & 53.04 & 4.77 & 5.05 & 2.46 \\
\hline 76 & 56 & $(30-60)$ & 0.34 & 0.4 & Type 1 & 1:1.17:1.93 & 60 & 51.71 & 4.83 & 5.07 & 2.44 \\
\hline 77 & 25 & $(10-30)$ & 0.34 & 0.4 & Type 1 & $1: 1.26: 2.45$ & 90 & \begin{tabular}{|l|}
49.50 \\
\end{tabular} & 4.89 & 5.09 & 2.42 \\
\hline
\end{tabular}


Table 1. Continued.

\begin{tabular}{|c|c|c|c|c|c|c|c|c|c|c|c|}
\hline $\begin{array}{c}\text { Sample } \\
\text { no. }\end{array}$ & $\begin{array}{c}\text { SLUMP } \\
(\mathbf{m m})\end{array}$ & $\begin{array}{c}\text { SLUMP } \\
\text { range } \\
(\mathrm{mm})\end{array}$ & $\begin{array}{c}\mathrm{SO}_{3} \% \\
\text { in fine } \\
\text { agregate }\end{array}$ & $\mathbf{W} / \mathbf{C}$ & $\begin{array}{c}\text { Coarse } \\
\text { Aggregate }\end{array}$ & $\begin{array}{c}\text { Mix } \\
\text { proportions }\end{array}$ & $\begin{array}{l}\text { Age } \\
\text { (day) }\end{array}$ & $\begin{array}{c}\text { Comp. } \\
\text { str. } \\
\text { (Mpa) }\end{array}$ & $\begin{array}{c}\text { Ult. } \\
\mathrm{V}(\mathrm{km} / \mathrm{s}) \\
\text { direct }\end{array}$ & $\begin{array}{c}\text { Ult. } \\
\text { V(km/s)s } \\
\text { urface }\end{array}$ & $\begin{array}{c}\text { Density } \\
(\mathrm{gm} / \mathrm{cm} 3)\end{array}$ \\
\hline 78 & 25 & $(10-30)$ & 0.34 & 0.4 & Type 1 & $1: 1.26: 2.45$ & 100 & 34.47 & & 4.55 & \\
\hline 79 & 8 & $(0-10)$ & 0.34 & 0.45 & Type 2 & 1:1.6:3.4 & 7 & 24.11 & 4.61 & 4.73 & 2.45 \\
\hline 80 & 8 & $(0-10)$ & 0.34 & 0.45 & Type 2 & 1:1.6:3.4 & 14 & 34.82 & 4.81 & 4.99 & 2.46 \\
\hline 81 & 8 & $(0-10)$ & 0.34 & 0.45 & Type 2 & 1:1.6:3.4 & 21 & 37.95 & 4.87 & 5.11 & 2.48 \\
\hline 82 & 8 & $(0-10)$ & 0.34 & 0.45 & Type 2 & 1:1.6:3.4 & 28 & 41.52 & 4.92 & 5.18 & 2.46 \\
\hline 83 & 8 & $(0-10)$ & $\mathbf{0 . 3 4}$ & 0.45 & Type 2 & 1:1.6:3.4 & 60 & 53.13 & 4.95 & 5.25 & 2.46 \\
\hline 84 & 8 & $(0-10)$ & 0.34 & 0.45 & Type 2 & 1:1.6:3.4 & 90 & 53.57 & 5.00 & 5.23 & 2.45 \\
\hline 85 & 8 & $(0-10)$ & $\mathbf{0 . 3 4}$ & 0.45 & Type 2 & 1:1.6:3.4 & 120 & 52.68 & 5.01 & 5.20 & 2.43 \\
\hline 86 & 70 & \begin{tabular}{|l|}
$(60-180)$ \\
\end{tabular} & 2.05 & 0.48 & Type 1 & \begin{tabular}{|l|l|}
$1: 32: 2.18$ \\
\end{tabular} & 28 & 26.12 & 4.51 & 4.55 & \\
\hline 87 & 70 & $(60-180)$ & 2.05 & 0.48 & Type 1 & 1:1.32:2.18 & 7 & 22.32 & 4.34 & 3.90 & 2.38 \\
\hline 88 & 70 & \begin{tabular}{|l|}
$(60-180)$ \\
\end{tabular} & 2.05 & 0.48 & Type 1 & 1:1.32:2.18 & 28 & 40.40 & 4.66 & 4.70 & 2.36 \\
\hline 89 & 70 & \begin{tabular}{|l|}
$(60-180)$ \\
\end{tabular} & 2.05 & 0.48 & Type 1 & $1: 1.32: 2.18$ & 60 & 39.29 & 4.67 & 4.76 & 2.43 \\
\hline 90 & 105 & \begin{tabular}{|l|}
$(60-180)$ \\
\end{tabular} & $\mathbf{0 . 3 4}$ & 0.5 & Type 3 & \begin{tabular}{|l|l|}
$1: 1.71: 1.93$ \\
\end{tabular} & 7 & 33.59 & 4.46 & 4.68 & 2.38 \\
\hline 91 & 105 & $(60-180)$ & 0.34 & 0.5 & Type 3 & 1:1.71:1.93 & 14 & 38.45 & 4.55 & 4.79 & 2.39 \\
\hline 92 & 105 & \begin{tabular}{|c|}
$(60-180)$ \\
\end{tabular} & 0.34 & 0.5 & Type 3 & 1:1.71:1.93 & 21 & 38.45 & 4.59 & 4.83 & 2.39 \\
\hline 93 & 105 & \begin{tabular}{|c|}
$(60-180)$ \\
\end{tabular} & 0.34 & 0.5 & Type 3 & 1:1.71:1.93 & 28 & 42.87 & 4.58 & 4.89 & 2.41 \\
\hline 94 & 105 & \begin{tabular}{|c|}
$(60-180)$ \\
\end{tabular} & 0.34 & 0.5 & Type 3 & $1: 1.71: 1.93$ & 60 & 45.52 & 4.63 & 4.94 & 2.37 \\
\hline 95 & 105 & \begin{tabular}{|c|}
$(60-180)$ \\
\end{tabular} & 0.34 & 0.5 & Type 3 & $1: 1.71: 1.93$ & 90 & 46.41 & 4.63 & 4.94 & 2.39 \\
\hline 96 & 105 & \begin{tabular}{|l|}
$(60-180)$ \\
\end{tabular} & 0.34 & 0.5 & Type 3 & $1: 1.71: 1.93$ & 150 & 50.38 & 4.68 & 4.96 & 2.39 \\
\hline 97 & 65 & \begin{tabular}{|l}
$(60-180)$ \\
\end{tabular} & 0.34 & 0.48 & Type 1 & $1: 1.32: 2.18$ & 7 & 20.98 & 4.51 & 4.21 & 2.38 \\
\hline 98 & 65 & \begin{tabular}{|l|}
$(60-180)$ \\
\end{tabular} & 0.34 & 0.48 & Type 1 & $1: 1.32: 2.18$ & 28 & 29.46 & 4.67 & 4.62 & 2.39 \\
\hline 99 & 65 & \begin{tabular}{|c|}
$(60-180)$ \\
\end{tabular} & $\mathbf{0 . 3 4}$ & 0.48 & Type 1 & $1: 1.32: 2.18$ & 60 & 35.49 & 4.66 & 4.71 & 2.40 \\
\hline 100 & 65 & \begin{tabular}{|c|}
$(60-180)$ \\
\end{tabular} & 0.34 & 0.48 & Type 1 & $1: 1.32: 2.18$ & 90 & 41.52 & 4.69 & 4.67 & 2.42 \\
\hline 101 & 65 & \begin{tabular}{|c|}
$(60-180)$ \\
\end{tabular} & 0.34 & 0.48 & Type 1 & $1: 1.32: 2.18$ & 120 & 25.45 & 4.70 & 4.65 & 2.48 \\
\hline 102 & 65 & \begin{tabular}{|c|}
$(60-180)$ \\
\end{tabular} & 0.34 & 0.48 & Type 1 & $1: 1.32: 2.18$ & 150 & 35.27 & 4.92 & 5.22 & 2.49 \\
\hline 103 & 9 & \begin{tabular}{|l|}
$(0-10)$ \\
\end{tabular} & 2.05 & 0.5 & Type 2 & 1:1.69:4.82 & 7 & 25.89 & 4.78 & 4.11 & 2.31 \\
\hline 104 & 9 & $(0-10)$ & 2.05 & 0.5 & Type 2 & $1: 1.69: 4.82$ & 28 & \begin{tabular}{|l|}
30.36 \\
\end{tabular} & 4.89 & 5.03 & 2.47 \\
\hline 105 & 9 & $(0-10)$ & 2.05 & 0.5 & Type 2 & 1:1.69:4.82 & 60 & 38.39 & 4.90 & 5.28 & 2.48 \\
\hline 106 & 9 & $(0-10)$ & 2.05 & 0.5 & Type 2 & $1: 1.69: 4.82$ & 90 & 38.39 & 4.97 & 5.18 & 2.48 \\
\hline 107 & 9 & $(0-10)$ & 2.05 & 0.5 & Type 2 & $1: 1.69: 4.82$ & 120 & 32.14 & 5.01 & 5.18 & 2.48 \\
\hline 108 & 15 & $(10-30)$ & 2.05 & 0.5 & Type 2 & $1: 1.52: 3.92$ & 7 & 16.96 & 4.47 & 4.42 & 2.43 \\
\hline 109 & 15 & \begin{tabular}{|c|}
$(10-30)$ \\
\end{tabular} & 2.05 & 0.5 & Type 2 & $1: 1.52: 3.92$ & 28 & 22.32 & 4.70 & 4.69 & 2.42 \\
\hline 110 & 15 & $(10-30)$ & 2.05 & 0.5 & Type 2 & $1: 1.52: 3.92$ & 60 & 30.36 & 4.71 & 4.72 & 2.41 \\
\hline 111 & 15 & $(10-30)$ & 2.05 & 0.5 & Type 2 & $1: 1.52: 3.92$ & 90 & 39.29 & 4.71 & 4.70 & 2.40 \\
\hline 112 & 15 & $(10-30)$ & 2.05 & 0.5 & Type 2 & $1: 1.52: 3.92$ & 120 & 36.16 & 4.75 & 4.71 & 2.40 \\
\hline 113 & 45 & \begin{tabular}{|c|}
$(30-60)$ \\
\end{tabular} & 2.05 & 0.5 & Type 2 & $1: 1.39: 3.26$ & 7 & 21.65 & 4.46 & 4.59 & 2.38 \\
\hline 114 & 45 & $(30-60)$ & 2.05 & 0.5 & Type 2 & $1: 1.39: 3.26$ & 28 & 30.36 & 4.64 & 4.91 & 2.43 \\
\hline 115 & 45 & \begin{tabular}{|c|}
$(30-60)$ \\
\end{tabular} & 2.05 & 0.5 & Type 2 & $1: 1.39: 3.26$ & 60 & 29.02 & 4.69 & 4.96 & 2.43 \\
\hline 116 & 45 & \begin{tabular}{|c|}
$(30-60)$ \\
\end{tabular} & 2.05 & 0.5 & Type 2 & 1:1.39:3.26 & 90 & 38.17 & 4.71 & 4.97 & 2.40 \\
\hline 117 & 45 & \begin{tabular}{|l|}
$(30-60)$ \\
\end{tabular} & 2.05 & 0.5 & Type 2 & $1: 1.39: 3.26$ & 120 & 37.05 & 4.74 & 4.96 & 2.40 \\
\hline 118 & 85 & \begin{tabular}{|c|}
$(60-180)$ \\
\end{tabular} & 2.05 & 0.5 & Type 2 & 1:1.42:2.75 & 7 & 22.32 & 4.44 & 4.01 & 2.42 \\
\hline 119 & 85 & \begin{tabular}{|l|}
$(60-180)$ \\
\end{tabular} & 2.05 & 0.5 & Type 2 & $1: 1.42: 2.75$ & 14 & 33.71 & 4.75 & 4.51 & 2.41 \\
\hline 120 & 85 & \begin{tabular}{|c|}
$(60-180)$ \\
\end{tabular} & 2.05 & 0.5 & Type 2 & $1: 1.42: 2.75$ & 21 & 28.57 & 4.74 & 4.97 & 2.41 \\
\hline 121 & 85 & \begin{tabular}{|l|}
$(60-180)$ \\
\end{tabular} & 2.05 & 0.5 & Type 2 & $1: 1.42: 2.75$ & 28 & 32.81 & 4.76 & 5.00 & 2.44 \\
\hline 122 & 85 & \begin{tabular}{|l|}
$(60-180)$ \\
\end{tabular} & 2.05 & 0.5 & Type 2 & $1: 1.42: 2.75$ & 60 & 38.84 & 4.77 & 5.10 & 2.43 \\
\hline 123 & 85 & \begin{tabular}{|l|}
$(60-180)$ \\
\end{tabular} & 2.05 & 0.5 & Type 2 & 1:1.42:2.75 & 90 & 41.07 & 4.81 & 5.11 & 2.39 \\
\hline 124 & 85 & \begin{tabular}{|c|}
$(60-180)$ \\
\end{tabular} & 2.05 & 0.5 & Type 2 & $1: 1.42: 2.75$ & 120 & 49.55 & 4.83 & 5.08 & 2.41 \\
\hline 125 & 20 & \begin{tabular}{|c|}
$(10-30)$ \\
\end{tabular} & 0.34 & 0.5 & Type 2 & $1: 2.37: 3.87$ & 7 & 31.25 & 4.76 & 4.94 & 2.39 \\
\hline 126 & 20 & \begin{tabular}{|c|}
$(10-30)$ \\
\end{tabular} & 0.34 & 0.5 & Type 2 & 1:2.37:3.87 & 14 & 33.04 & 4.84 & 5.11 & 2.41 \\
\hline 127 & 20 & $(\mathbf{1 0 - 3 0})$ & 0.34 & 0.5 & Type 2 & $1: 2.37: 3.87$ & 21 & 35.71 & 4.87 & 5.14 & 2.42 \\
\hline 128 & 20 & \begin{tabular}{|c|}
$(10-30)$ \\
\end{tabular} & 0.34 & 0.5 & Type 2 & $1: 2.37: 3.87$ & 28 & 40.40 & 4.91 & 5.19 & 2.42 \\
\hline 129 & 20 & \begin{tabular}{|c|}
$(10-30)$ \\
\end{tabular} & 0.34 & 0.5 & Type 2 & $1: 2.37: 3.87$ & 60 & 42.86 & 4.98 & 5.25 & 2.42 \\
\hline 130 & 20 & \begin{tabular}{|c|}
$(10-30)$ \\
\end{tabular} & 0.34 & 0.5 & Type 2 & $1: 2.37: 3.87$ & 90 & 49.11 & 4.97 & 5.27 & 2.42 \\
\hline 131 & 20 & \begin{tabular}{|c|}
$(10-30)$ \\
\end{tabular} & 0.34 & 0.5 & Type 2 & $1: 2.37: 3.87$ & 120 & 46.43 & 4.95 & 5.26 & 2.42 \\
\hline 132 & 20 & \begin{tabular}{|l|}
$(10-30)$ \\
\end{tabular} & 0.34 & 0.5 & Type 2 & $1: 2.37: 3.87$ & 150 & 43.30 & 4.98 & 5.27 & 2.42 \\
\hline 133 & 77 & \begin{tabular}{|c|}
$(60-180)$ \\
\end{tabular} & 0.34 & 0.4 & Type 1 & 1:1.13:1.7 & 14 & 32.24 & 4.72 & 4.95 & 2.33 \\
\hline 134 & 77 & $(60-180)$ & 0.34 & 0.4 & Type 1 & 1:1.13:1.7 & 21 & 44.19 & 4.79 & 5.05 & 2.35 \\
\hline 135 & 77 & \begin{tabular}{|c|}
$(60-180)$ \\
\end{tabular} & 0.34 & 0.4 & Type 1 & 1:1.13:1.7 & 28 & \begin{tabular}{|l|}
43.74 \\
\end{tabular} & 4.80 & 5.07 & 2.35 \\
\hline 136 & 77 & $(60-180)$ & 0.34 & 0.4 & Type 1 & 1:1.13:1.7 & 60 & 49.15 & 4.89 & 5.13 & 2.36 \\
\hline 137 & 77 & \begin{tabular}{|c|}
$(60-180)$ \\
\end{tabular} & 0.34 & 0.4 & Type 1 & 1:1.13:1.7 & 90 & 46.89 & 4.88 & 5.09 & 2.35 \\
\hline 138 & 58 & \begin{tabular}{|c|}
$(30-60)$ \\
\end{tabular} & 0.34 & 0.5 & Type 2 & $1: 1.9: 2.74$ & 7 & 29.24 & 4.61 & 4.75 & 2.34 \\
\hline 139 & 58 & \begin{tabular}{|c|}
$(30-60)$ \\
\end{tabular} & 0.34 & 0.5 & Type 2 & $1: 1.9: 2.74$ & 14 & 35.71 & 4.77 & 4.92 & 2.36 \\
\hline 140 & 58 & \begin{tabular}{|c|}
$(30-60)$ \\
\end{tabular} & 0.34 & 0.5 & Type 2 & $1: 1.9: 2.74$ & 21 & 41.07 & 4.78 & 5.00 & 2.37 \\
\hline 141 & 58 & \begin{tabular}{|c|}
$(30-60)$ \\
\end{tabular} & 0.34 & 0.5 & Type 2 & $1: 1.9: 2.74$ & 28 & 38.39 & 4.82 & 5.08 & 2.37 \\
\hline 142 & 58 & \begin{tabular}{|c|}
$(30-60)$ \\
\end{tabular} & 0.34 & 0.5 & Type 2 & $1: 1.9: 2.74$ & 60 & 41.96 & 4.84 & 5.10 & 2.37 \\
\hline 143 & 58 & \begin{tabular}{|c|}
$(30-60)$ \\
\end{tabular} & 0.34 & 0.5 & Type 2 & $1: 1.9: 2.74$ & 90 & 52.68 & 4.89 & 5.13 & 2.36 \\
\hline 144 & 58 & \begin{tabular}{|c|}
$(30-60)$ \\
\end{tabular} & 0.34 & 0.5 & Type 2 & $1: 1.9: 2.74$ & 120 & 46.88 & 4.89 & 5.10 & 2.36 \\
\hline 145 & 58 & \begin{tabular}{|c|}
$(30-60)$ \\
\end{tabular} & 0.34 & 0.5 & Type 2 & $1: 1.9: 2.74$ & 150 & 41.07 & 4.91 & 5.12 & 2.36 \\
\hline 146 & 72 & \begin{tabular}{|c|}
$(60-180)$ \\
\end{tabular} & 0.34 & 0.5 & Type 2 & 1:1.91:2.25 & 7 & 28.35 & 4.68 & 4.81 & 2.36 \\
\hline 147 & 72 & \begin{tabular}{|l|}
$(60-180)$ \\
\end{tabular} & 0.34 & 0.5 & Type 2 & $1: 1.91: 2.25$ & 14 & 29.91 & 4.74 & 4.93 & 2.33 \\
\hline 148 & 72 & \begin{tabular}{|c|}
$(60-180)$ \\
\end{tabular} & 0.34 & 0.5 & Type 2 & 1:1.91:2.25 & 21 & 31.70 & 4.80 & 4.96 & 2.38 \\
\hline 149 & 72 & \begin{tabular}{|l|}
$(60-180)$ \\
\end{tabular} & 0.34 & 0.5 & Type 2 & $1: 1.91: 2.25$ & 28 & 38.84 & 4.80 & 5.03 & 2.39 \\
\hline 150 & 72 & \begin{tabular}{|c|}
$(60-180)$ \\
\end{tabular} & 0.34 & 0.5 & Type 2 & $1: 1.91: 2.25$ & 60 & 43.75 & 4.85 & 5.06 & 2.38 \\
\hline 151 & 72 & \begin{tabular}{|l|}
$(60-180)$ \\
\end{tabular} & 0.34 & 0.5 & Type 2 & 1:1.91:2.25 & 90 & 40.63 & 4.87 & 5.08 & 2.37 \\
\hline 152 & 72 & \begin{tabular}{|l|}
$(60-180)$ \\
\end{tabular} & 0.34 & 0.5 & Type 2 & $1: 1.91: 2.25$ & 120 & 44.20 & 4.87 & 5.08 & 2.37 \\
\hline 153 & 72 & \begin{tabular}{|c|}
$(60-180)$ \\
\end{tabular} & 0.34 & 0.5 & Type 2 & $1: 1.91: 2.25$ & 150 & 44.20 & 4.90 & 5.10 & 2.38 \\
\hline 154 & 72 & \begin{tabular}{|l|}
$(60-180)$ \\
\end{tabular} & 0.34 & 0.5 & Type 2 & $1: 1.91: 2.25$ & 120 & 39.78 & 4.90 & 5.07 & 2.37 \\
\hline
\end{tabular}


Table 1. Continued.

\begin{tabular}{|c|c|c|c|c|c|c|c|c|c|c|c|}
\hline $\begin{array}{c}\text { Sample } \\
\text { no. }\end{array}$ & $\begin{array}{c}\text { SLUMP } \\
(\mathbf{m m})\end{array}$ & $\begin{array}{c}\text { SLUMP } \\
\text { range } \\
(\mathbf{m m})\end{array}$ & $\begin{array}{c}\mathrm{SO}_{3} \% \text { in } \\
\text { fine } \\
\text { agregate }\end{array}$ & W/C & $\begin{array}{c}\text { Coarse } \\
\text { Aggregate }\end{array}$ & $\begin{array}{c}\text { Mix } \\
\text { proportions }\end{array}$ & $\begin{array}{l}\text { Age } \\
\text { (day) }\end{array}$ & $\begin{array}{c}\text { Comp. } \\
\text { str. } \\
\text { (Mpa) }\end{array}$ & $\begin{array}{c}\text { Ult. } \\
\text { V(km/s) } \\
\text { direct }\end{array}$ & $\begin{array}{c}\text { Ult. } \\
\text { V(km/s)su } \\
\text { rface }\end{array}$ & $\begin{array}{c}\text { Density } \\
\text { (gm /cm3) }\end{array}$ \\
\hline 155 & 72 & \begin{tabular}{|c|}
$(60-180)$ \\
\end{tabular} & 0.34 & 0.5 & Type 5 & 1:1.91:2.25 & 7 & 23.20 & 4.56 & 4.71 & 2.35 \\
\hline 156 & 72 & \begin{tabular}{|c|}
$(60-180)$ \\
\end{tabular} & 0.34 & 0.5 & Type 5 & 1:1.91:2.25 & 41 & 35.36 & 4.71 & 4.92 & 2.37 \\
\hline 157 & 72 & \begin{tabular}{|c|}
$(60-180)$ \\
\end{tabular} & 0.34 & 0.5 & Type 5 & $1: 1.91: 2.25$ & 21 & 35.80 & 4.76 & 4.95 & 2.36 \\
\hline 158 & 72 & \begin{tabular}{|c|}
$(60-180)$ \\
\end{tabular} & 0.34 & 0.5 & Type 5 & $1: 1.91: 2.25$ & 28 & 38.89 & 4.79 & 5.03 & 2.34 \\
\hline 159 & 72 & \begin{tabular}{|c|}
$(60-180)$ \\
\end{tabular} & 0.34 & 0.5 & Type 5 & 1:1.91:2.25 & 60 & 45.74 & 4.84 & 5.08 & 2.36 \\
\hline 160 & 72 & \begin{tabular}{|c|}
$(60-180)$ \\
\end{tabular} & 0.34 & 0.5 & Type 5 & 1:1.91:2.25 & 90 & 52.59 & 4.87 & 5.12 & 2.35 \\
\hline 161 & 92 & \begin{tabular}{|l|}
$(60-180)$ \\
\end{tabular} & 0.34 & 0.5 & Type 4 & 1:1.71:1.93 & 7 & 33.93 & 4.51 & 4.73 & 2.38 \\
\hline 162 & 92 & \begin{tabular}{|c|}
$(60-180)$ \\
\end{tabular} & 0.34 & 0.5 & Type 4 & 1:1.71:1.93 & 14 & 38.84 & 4.59 & 4.84 & 2.39 \\
\hline 163 & 92 & \begin{tabular}{|c|}
$(60-180)$ \\
\end{tabular} & 0.34 & 0.5 & Type 4 & 1:1.71:1.93 & 21 & 38.84 & 4.63 & 4.88 & 2.39 \\
\hline 164 & 92 & \begin{tabular}{|c|}
$(60-180)$ \\
\end{tabular} & 0.34 & 0.5 & Type 4 & 1:1.71:1.93 & 28 & 43.30 & 4.63 & 4.94 & 2.41 \\
\hline 165 & 92 & \begin{tabular}{|c|}
$(60-180)$ \\
\end{tabular} & 0.34 & 0.5 & Type 4 & 1:1.71:1.93 & 60 & 45.98 & 4.68 & 4.99 & 2.37 \\
\hline 166 & 92 & \begin{tabular}{|l|}
$(60-180)$ \\
\end{tabular} & 0.34 & 0.5 & Type 4 & 1:1.71:1.93 & 90 & 46.88 & 4.68 & 4.99 & 2.39 \\
\hline 167 & 92 & \begin{tabular}{|c|}
$(60-180)$ \\
\end{tabular} & 0.34 & 0.5 & Type 4 & 1:1.71:1.93 & 120 & 46.88 & 4.70 & 4.99 & 2.39 \\
\hline 168 & 92 & \begin{tabular}{|c|}
$(60-180)$ \\
\end{tabular} & 0.34 & 0.5 & Type 4 & 1:1.71:1.93 & 150 & 50.89 & 4.73 & 5.01 & 2.39 \\
\hline 169 & 98 & \begin{tabular}{|c|}
$(60-180)$ \\
\end{tabular} & 2.05 & 0.5 & Type 4 & $1: 1.24: 2.412$ & 7 & 14.51 & 4.01 & 3.95 & 2.33 \\
\hline 170 & 98 & \begin{tabular}{|c|}
$(60-180)$ \\
\end{tabular} & 2.05 & 0.5 & Type 4 & $1: 1.24: 2.412$ & 14 & 17.86 & 4.24 & 4.38 & 2.33 \\
\hline 171 & 98 & \begin{tabular}{|c|}
$(60-180)$ \\
\end{tabular} & 2.05 & 0.5 & Type 4 & $1: 1.24: 2.412$ & 21 & 22.77 & 4.32 & 4.46 & 2.33 \\
\hline 172 & 98 & \begin{tabular}{|l|}
$(60-180)$ \\
\end{tabular} & 2.05 & 0.5 & Type 4 & $1: 1.24: 2.412$ & 28 & 23.66 & 4.39 & 4.58 & 2.33 \\
\hline 173 & 98 & \begin{tabular}{|c|}
$(60-180)$ \\
\end{tabular} & 2.05 & 0.5 & Type 4 & $1: 1.24: 2.412$ & 60 & 32.14 & 4.45 & 4.65 & 2.66 \\
\hline 174 & 98 & \begin{tabular}{|c|}
$(60-180)$ \\
\end{tabular} & 2.05 & 0.5 & Type 4 & $1: 1.24: 2.412$ & 90 & 32.14 & 4.47 & 4.68 & 2.31 \\
\hline 175 & 98 & \begin{tabular}{|c|}
$(60-180)$ \\
\end{tabular} & 2.05 & 0.5 & Type 4 & $1: 1.24: 2.412$ & 120 & 30.80 & 4.52 & 4.72 & 2.31 \\
\hline 176 & 85 & \begin{tabular}{|c|}
$(60-180)$ \\
\end{tabular} & 0.34 & 0.5 & Type 1 & $1: 1.75: 2.63$ & 7 & 19.84 & 4.72 & 4.75 & 2.39 \\
\hline 177 & 85 & \begin{tabular}{|c|}
$(60-180)$ \\
\end{tabular} & 0.34 & 0.5 & Type 1 & $1: 1.75: 2.63$ & 14 & 27.96 & 4.81 & 5.04 & 2.43 \\
\hline 178 & 85 & \begin{tabular}{|c|}
$(60-180)$ \\
\end{tabular} & 0.34 & 0.5 & Type 1 & $1: 1.75: 2.63$ & 21 & 29.31 & 4.87 & 5.10 & 2.42 \\
\hline 179 & 85 & \begin{tabular}{|c|}
$(60-180)$ \\
\end{tabular} & 0.34 & 0.5 & Type 1 & $1: 1.75: 2.63$ & 28 & 35.62 & 4.94 & 5.13 & 2.43 \\
\hline 180 & 85 & \begin{tabular}{|c|}
$(60-180)$ \\
\end{tabular} & 0.34 & 0.5 & Type 1 & $1: 1.75: 2.63$ & 60 & 41.48 & 4.96 & 5.17 & 2.41 \\
\hline 181 & 85 & \begin{tabular}{|c|}
$(60-180)$ \\
\end{tabular} & 0.34 & 0.5 & Type 1 & 1:1.75:2.63 & 90 & 41.93 & 4.98 & 5.14 & 2.41 \\
\hline 182 & 70 & \begin{tabular}{|c|}
$(60-180)$ \\
\end{tabular} & 0.34 & 0.5 & Type 5 & $1: 1.91: 2.25$ & 7 & 23.44 & 4.61 & 4.76 & 2.35 \\
\hline 183 & 70 & \begin{tabular}{|c|}
$(60-180)$ \\
\end{tabular} & 0.34 & 0.5 & Type 5 & $1: 1.91: 2.25$ & 41 & 35.71 & 4.76 & 4.97 & 2.37 \\
\hline 184 & 70 & \begin{tabular}{|c|}
$(60-180)$ \\
\end{tabular} & 0.34 & 0.5 & Type 5 & $1: 1.91: 2.25$ & 21 & 36.16 & 4.81 & 5.00 & 2.36 \\
\hline 185 & 70 & \begin{tabular}{|l|}
$(60-180)$ \\
\end{tabular} & 0.34 & 0.5 & Type 5 & 1:1.91:2.25 & 28 & 39.29 & 4.84 & 5.08 & 2.34 \\
\hline 186 & 70 & \begin{tabular}{|c|}
$(60-180)$ \\
\end{tabular} & 0.34 & 0.5 & Type 5 & $1: 1.91: 2.25$ & 60 & 46.21 & 4.89 & 5.13 & 2.36 \\
\hline 187 & 70 & \begin{tabular}{|c|}
$(60-180)$ \\
\end{tabular} & 0.34 & 0.5 & Type 5 & $1: 1.91: 2.25$ & 90 & 53.13 & 4.92 & 5.17 & 2.35 \\
\hline 188 & 70 & \begin{tabular}{|l|}
$(60-180)$ \\
\end{tabular} & 0.34 & 0.5 & Type 5 & 1:1.91:2.25 & 120 & 40.18 & 4.95 & 5.12 & 2.37 \\
\hline 189 & 90 & \begin{tabular}{|c|}
$(60-180)$ \\
\end{tabular} & 2.05 & 0.5 & Type 5 & 1:1.41:2.75 & 7 & 8.93 & 4.13 & 3.47 & 2.40 \\
\hline 190 & 90 & \begin{tabular}{|c|}
$(60-180)$ \\
\end{tabular} & 2.05 & 0.5 & Type 5 & 1:1.41:2.75 & 14 & 13.84 & 4.24 & 3.66 & 2.37 \\
\hline 191 & 90 & \begin{tabular}{|c|}
$(60-180)$ \\
\end{tabular} & 2.05 & 0.5 & Type 5 & 1:1.41:2.75 & 21 & 12.95 & 4.32 & 4.03 & 2.40 \\
\hline 192 & 90 & \begin{tabular}{|l|}
$(60-180)$ \\
\end{tabular} & 2.05 & 0.5 & Type 5 & 1:1.41:2.75 & 28 & 13.84 & 4.38 & 4.06 & 2.42 \\
\hline 193 & 90 & \begin{tabular}{|c}
$(60-180)$ \\
\end{tabular} & 2.05 & 0.5 & Type 5 & $1: 1.41: 2.75$ & 60 & 20.54 & 4.44 & 4.13 & 2.38 \\
\hline 194 & 90 & \begin{tabular}{|c|}
$(60-180)$ \\
\end{tabular} & 2.05 & 0.5 & Type 5 & $1: 1.41: 2.75$ & 90 & 22.77 & 4.50 & 4.46 & 2.35 \\
\hline 195 & 90 & \begin{tabular}{|l|}
$(60-180)$ \\
\end{tabular} & 2.05 & 0.5 & Type 5 & 1:1.41:2.75 & 120 & 21.43 & 4.51 & 4.46 & 2.33 \\
\hline 196 & 10 & \begin{tabular}{|c|}
$(0-10)$ \\
\end{tabular} & 0.34 & 0.5 & Type 1 & $1: 1.82: 4.21$ & 14 & 24.55 & 5.01 & 5.14 & 2.45 \\
\hline 197 & 10 & $(0-10)$ & 0.34 & 0.5 & Type 1 & $1: 1.82: 4.21$ & 21 & 31.70 & 5.11 & 5.25 & 2.49 \\
\hline 198 & 10 & $(0-10)$ & 0.34 & 0.5 & Type 1 & $1: 1.82: 4.21$ & 28 & 34.38 & 5.10 & 5.28 & 2.46 \\
\hline 199 & 10 & $(0-10)$ & 0.34 & 0.5 & Type 1 & $1: 1.82: 4.21$ & 60 & 40.63 & 5.14 & 5.32 & 2.47 \\
\hline 200 & 10 & $(0-10)$ & 0.34 & 0.5 & Type 1 & $1: 1.82: 4.21$ & 90 & 39.29 & 5.19 & 5.30 & 2.46 \\
\hline 201 & 27 & $(10-30)$ & 0.34 & 0.5 & Type 1 & $1: 1.76: 3.74$ & 7 & 21.88 & 4.64 & 4.81 & 2.43 \\
\hline 202 & 27 & $(10-30)$ & 0.34 & 0.5 & Type 1 & $1: 1.76: 3.74$ & 14 & 30.36 & 4.80 & 5.04 & 2.45 \\
\hline 203 & 27 & $(10-30)$ & 0.34 & 0.5 & Type 1 & $1: 1.76: 3.74$ & 21 & 32.59 & 4.89 & 5.11 & 2.45 \\
\hline 204 & 27 & $(10-30)$ & 0.34 & 0.5 & Type 1 & $1: 1.76: 3.74$ & 28 & 41.52 & 4.91 & 5.15 & 2.44 \\
\hline 205 & 27 & $(10-30)$ & 0.34 & 0.5 & Type 1 & $1: 1.76: 3.74$ & 60 & 38.39 & 4.91 & 5.15 & 2.44 \\
\hline 206 & 75 & \begin{tabular}{|c|}
$(60-180)$ \\
\end{tabular} & 0.34 & 0.45 & Type 2 & 1:1.47:1.86 & 120 & 45.96 & 4.70 & 4.94 & 2.36 \\
\hline 207 & 75 & \begin{tabular}{|c|}
$(60-180)$ \\
\end{tabular} & 0.34 & 0.45 & Type 2 & 1:1.47:1.86 & 7 & 34.03 & 4.42 & 4.62 & 2.37 \\
\hline 208 & 75 & \begin{tabular}{|c|}
$(60-180)$ \\
\end{tabular} & 0.34 & 0.45 & Type 2 & 1:1.47:1.86 & 14 & 33.15 & 4.53 & 4.74 & 2.37 \\
\hline 209 & 75 & \begin{tabular}{|c|}
$(60-180)$ \\
\end{tabular} & 0.34 & 0.45 & Type 2 & 1:1.47:1.86 & 21 & 34.27 & 4.67 & 4.94 & 2.38 \\
\hline 210 & 75 & \begin{tabular}{|c|}
$(60-180)$ \\
\end{tabular} & 0.34 & 0.45 & Type 2 & 1:1.47:1.86 & 28 & 39.68 & 4.69 & 4.98 & 2.37 \\
\hline 211 & 75 & \begin{tabular}{|c|}
$(60-180)$ \\
\end{tabular} & 0.34 & 0.45 & Type 2 & 1:1.47:1.86 & 60 & 45.09 & 4.76 & 5.07 & 2.36 \\
\hline 212 & 75 & \begin{tabular}{|c|}
$(60-180)$ \\
\end{tabular} & 0.34 & 0.45 & Type 2 & 1:1.47:1.86 & 90 & 46.89 & 4.81 & 5.04 & 2.36 \\
\hline 213 & 55 & \begin{tabular}{|c|}
$(30-60)$ \\
\end{tabular} & 0.34 & 0.5 & Type 1 & 1:1.71:3.18 & 7 & 20.09 & 5.02 & 4.69 & 2.38 \\
\hline 214 & 55 & $(30-60)$ & 0.34 & 0.5 & Type 1 & 1:1.71:3.18 & 14 & 27.23 & 4.83 & 4.92 & 2.42 \\
\hline 215 & 55 & $(30-60)$ & 0.34 & 0.5 & Type 1 & $1: 1.71: 3.18$ & 21 & 30.80 & 4.89 & 5.04 & 2.38 \\
\hline 216 & 55 & $(30-60)$ & 0.34 & 0.5 & Type 1 & $1: 1.71: 3.18$ & 28 & 33.48 & 4.91 & 5.11 & 2.40 \\
\hline 217 & 55 & $(30-60)$ & 0.34 & 0.5 & Type 1 & 1:1.71:3.18 & 60 & 41.96 & 4.92 & 5.12 & 2.39 \\
\hline 218 & 55 & $(30-60)$ & 0.34 & 0.5 & Type 1 & $1: 1.71: 3.18$ & 90 & 37.05 & 4.96 & 5.11 & 2.39 \\
\hline 219 & 85 & \begin{tabular}{|l|}
$(60-180)$ \\
\end{tabular} & 0.34 & 0.5 & Type 1 & $1: 1.75: 2.63$ & 7 & 19.64 & 4.67 & 4.70 & 2.39 \\
\hline 220 & 85 & \begin{tabular}{|c|}
$(60-180)$ \\
\end{tabular} & 0.34 & 0.5 & Type 1 & $1: 1.75: 2.63$ & 14 & 27.68 & 4.76 & 4.99 & 2.43 \\
\hline 221 & 85 & \begin{tabular}{|l|}
$(60-180)$ \\
\end{tabular} & 0.34 & 0.5 & Type 1 & $1: 1.75: 2.63$ & 21 & 29.02 & 4.82 & 5.05 & 2.42 \\
\hline 222 & 85 & \begin{tabular}{|c|}
$(60-180)$ \\
\end{tabular} & 0.34 & 0.5 & Type 1 & $1: 1.75: 2.63$ & 28 & 35.27 & 4.89 & 5.08 & 2.43 \\
\hline 223 & 85 & \begin{tabular}{|c|}
$(60-180)$ \\
\end{tabular} & 0.34 & 0.5 & Type 1 & $1: 1.75: 2.63$ & 60 & 41.07 & 4.91 & 5.12 & 2.41 \\
\hline 224 & 85 & \begin{tabular}{|c|}
$(60-180)$ \\
\end{tabular} & 0.34 & 0.5 & Type 1 & $1: 1.75: 2.63$ & 90 & 41.52 & 4.93 & 5.09 & 2.41 \\
\hline 225 & 5 & $(0-10)$ & 2.05 & 0.56 & Type 2 & 1:2.1:5.99 & 21 & 30.36 & 4.82 & 5.14 & 2.47 \\
\hline 226 & 5 & $(0-10)$ & 2.05 & 0.56 & Type 2 & $1: 2.1: 5.99$ & 28 & 29.91 & 4.89 & 5.18 & 2.47 \\
\hline 227 & 5 & $(0-10)$ & 2.05 & 0.56 & Type 2 & 1:2.1:5.99 & 60 & 35.71 & 4.92 & 5.26 & 2.48 \\
\hline 228 & 5 & $(0-10)$ & 2.05 & 0.56 & Type 2 & $1: 2.1: 5.99$ & 90 & 41.96 & 4.92 & 5.25 & 2.47 \\
\hline 229 & 5 & $(0-10)$ & 2.05 & 0.56 & Type 2 & $1: 2.1: 5.99$ & 120 & 42.41 & 4.93 & 5.27 & 2.47 \\
\hline 230 & 5 & $(0-10)$ & 2.05 & 0.56 & Type 2 & $1: 2.1: 5.99$ & 150 & 25.00 & 4.93 & 5.26 & 2.47 \\
\hline 231 & 115 & \begin{tabular}{|l|}
$(60-180)$ \\
\end{tabular} & 0.34 & 0.8 & Type 2 & $1: 3.33: 3.33$ & 7 & 5.26 & 3.73 & 3.06 & 2.34 \\
\hline
\end{tabular}


Table 1. Continued.

\begin{tabular}{|c|c|c|c|c|c|c|c|c|c|c|c|}
\hline $\begin{array}{c}\text { Sample } \\
\text { no. }\end{array}$ & $\begin{array}{c}\text { SLUMP } \\
(\mathbf{m m})\end{array}$ & $\begin{array}{c}\text { SLUMP } \\
\text { range } \\
(\mathbf{m m})\end{array}$ & $\begin{array}{c}\mathrm{SO}_{3} \% \text { in } \\
\text { fine } \\
\text { agregate }\end{array}$ & W/C & $\begin{array}{c}\text { Coarse } \\
\text { Aggregate }\end{array}$ & $\begin{array}{c}\text { Mix } \\
\text { proportions }\end{array}$ & $\begin{array}{l}\text { Age } \\
\text { (day) }\end{array}$ & $\begin{array}{l}\text { Comp. } \\
\text { str. } \\
\text { (Mpa) }\end{array}$ & $\begin{array}{c}\text { Ult. } \\
\text { V(km/s) } \\
\text { direct }\end{array}$ & $\begin{array}{c}\text { Ult. } \\
\mathbf{V}(\mathbf{k m} / \mathbf{s}) \mathbf{s u} \\
\text { rface }\end{array}$ & $\begin{array}{l}\text { Density } \\
(\mathrm{gm} / \mathrm{cm} 3)\end{array}$ \\
\hline 232 & 115 & $(60-180)$ & 0.34 & 0.8 & Type 2 & $1: 3.33: 3.33$ & 21 & 22.98 & 4.36 & 4.49 & 2.38 \\
\hline 233 & 115 & $(60-180)$ & 0.34 & 0.8 & Type 2 & 1:3.33:3.33 & 28 & 23.76 & 4.37 & 4.52 & 2.37 \\
\hline 234 & 115 & $(60-180)$ & 0.34 & 0.8 & Type 2 & $1: 3.33: 3.33$ & 60 & 25.95 & 4.44 & 4.61 & 2.36 \\
\hline 235 & 115 & $(60-180)$ & 0.34 & 0.8 & Type 2 & $1: 3.33: 3.33$ & 90 & 26.01 & 4.48 & 4.61 & 2.37 \\
\hline 236 & 20 & $(10-30)$ & 2.05 & 0.56 & Type 2 & $1: 2 .: 4.9$ & 7 & 11.61 & 4.42 & 4.07 & 2.43 \\
\hline 237 & 20 & $(10-30)$ & 2.05 & 0.56 & Type 2 & $1: 2 .: 4.9$ & 14 & 15.18 & 4.50 & 4.44 & 2.37 \\
\hline 238 & 20 & $(10-30)$ & 2.05 & 0.56 & Type 2 & $1: 2 .: 4.9$ & 21 & 16.07 & 4.57 & 4.58 & 2.37 \\
\hline 239 & 20 & $(10-30)$ & 2.05 & 0.56 & Type 2 & $1: 2 .: 4.9$ & 28 & 16.07 & 4.59 & 4.58 & 2.33 \\
\hline 240 & 20 & $(10-30)$ & 2.05 & 0.56 & Type 2 & $1: 2 .: 4.9$ & 60 & 19.20 & 4.65 & 4.71 & 2.36 \\
\hline 241 & 20 & $(10-30)$ & 2.05 & 0.56 & Type 2 & $1: 2 .: 4.9$ & 90 & 17.41 & 4.67 & 4.74 & 2.36 \\
\hline 242 & 20 & $(10-30)$ & 2.05 & 0.56 & Type 2 & $1: 2 .: 4.9$ & 120 & 26.79 & 4.75 & 4.79 & 2.36 \\
\hline 243 & 20 & $(10-30)$ & 2.05 & 0.56 & Type 2 & $1: 2 .: 4.9$ & 150 & 16.96 & 4.72 & 4.81 & 2.36 \\
\hline 244 & 25 & $(10-30)$ & 0.34 & 0.8 & Type 2 & 1:3.37:5.06 & 14 & 9.06 & 4.13 & 4.11 & 2.40 \\
\hline 245 & 25 & $(10-30)$ & 0.34 & 0.8 & Type 2 & 1:3.37:5.06 & 21 & 12.82 & 4.35 & 4.42 & 2.38 \\
\hline 246 & 25 & $(10-30)$ & 0.34 & 0.8 & Type 2 & 1:3.37:5.06 & 28 & 16.00 & 4.36 & 4.42 & 2.37 \\
\hline 247 & 25 & $(10-30)$ & 0.34 & 0.8 & Type 2 & 1:3.37:5.06 & 60 & 19.67 & 4.41 & 4.47 & 2.34 \\
\hline 248 & 25 & $(10-30)$ & 0.34 & 0.8 & Type 2 & 1:3.37:5.06 & 90 & 16.57 & 4.39 & 4.45 & 2.34 \\
\hline 249 & 35 & $(30-60)$ & 2.05 & 0.56 & Type 2 & 1:1.92:4.09 & 7 & 13.39 & 4.27 & 3.97 & 2.40 \\
\hline 250 & 35 & $(30-60)$ & 2.05 & 0.56 & Type 2 & 1:1.92:4.09 & 14 & 16.96 & 4.38 & 4.50 & 2.36 \\
\hline 251 & 35 & $(30-60)$ & 2.05 & 0.56 & Type 2 & 1:1.92:4.09 & 21 & 16.96 & 4.46 & 4.62 & 2.42 \\
\hline 252 & 35 & $(30-60)$ & 2.05 & 0.56 & Type 2 & 1:1.92:4.09 & 28 & 18.08 & 4.49 & 4.66 & 2.43 \\
\hline 253 & 35 & $(30-60)$ & 2.05 & 0.56 & Type 2 & 1:1.92:4.09 & 60 & 25.89 & 4.52 & 4.75 & 2.41 \\
\hline 254 & 35 & $(30-60)$ & 2.05 & 0.56 & Type 2 & 1:1.92:4.09 & 120 & 19.87 & 4.58 & 4.79 & 2.40 \\
\hline 255 & 110 & $(60-180)$ & 0.34 & 0.8 & Type 3 & $1: 3.33: 3.33$ & 7 & 6.76 & 3.70 & 3.03 & 2.34 \\
\hline 256 & 110 & $(60-180)$ & 0.34 & 0.8 & Type 3 & $1: 3.33: 3.33$ & 21 & 17.13 & 4.32 & 4.45 & 2.38 \\
\hline 257 & 110 & $(60-180)$ & 0.34 & 0.8 & Type 3 & $1: 3.33: 3.33$ & 28 & 17.58 & 4.33 & 4.48 & 2.37 \\
\hline 258 & 110 & $\begin{array}{l}(60-180) \\
\end{array}$ & 0.34 & 0.8 & Type 3 & $1: 3.33: 3.33$ & 60 & 21.19 & 4.40 & 4.56 & 2.36 \\
\hline 259 & 110 & $(60-180)$ & 0.34 & 0.8 & Type 3 & $1: 3.33: 3.33$ & 90 & 20.29 & 4.44 & 4.57 & 2.37 \\
\hline 260 & 70 & $(60-180)$ & 2.05 & 0.56 & Type 2 & $1: 1.96: 3.5$ & 7 & 12.95 & 4.13 & 3.93 & 2.61 \\
\hline 261 & 70 & $(60-180)$ & 2.05 & 0.56 & Type 2 & $1: 1.96: 3.5$ & 28 & 18.75 & 4.41 & 4.61 & 2.45 \\
\hline 262 & 70 & $(60-180)$ & 2.05 & 0.56 & Type 2 & $1: 1.96: 3.5$ & 60 & 23.21 & 4.44 & 4.71 & 2.44 \\
\hline 263 & 70 & $(60-180)$ & 2.05 & 0.56 & Type 2 & $1: 1.96: 3.5$ & 90 & 26.79 & 4.50 & 4.74 & 2.38 \\
\hline 264 & 70 & $(60-180)$ & 2.05 & 0.56 & Type 2 & $1: 1.96: 3.5$ & 120 & 20.54 & 4.53 & 4.73 & 2.42 \\
\hline 265 & 62 & $(60-180)$ & 0.34 & $\begin{array}{ll}0.4 \\
\end{array}$ & Type 1 & 1:1.13:1.7 & 14 & 31.28 & 4.58 & 4.80 & 2.33 \\
\hline 266 & 62 & $(60-180)$ & 0.34 & 0.4 & Type 1 & 1:1.13:1.7 & 21 & 42.88 & 4.65 & 4.90 & 2.35 \\
\hline 267 & 62 & $(60-180)$ & 0.34 & 0.4 & Type 1 & 1:1.13:1.7 & 28 & 42.44 & 4.85 & 5.12 & 2.35 \\
\hline 268 & 62 & $(60-180)$ & 0.34 & 0.4 & Type 1 & 1:1.13:1.7 & 60 & 47.69 & 4.94 & 5.18 & 2.36 \\
\hline 269 & 62 & $(60-180)$ & 0.34 & 0.4 & Type 1 & 1:1.13:1.7 & 14 & 31.28 & 4.77 & 4.99 & 2.33 \\
\hline 270 & 62 & $(60-180)$ & 0.34 & 0.4 & Type 1 & 1:1.13:1.7 & 21 & 42.88 & 4.84 & 5.10 & 2.35 \\
\hline 271 & 62 & $(60-180)$ & 0.34 & 0.4 & Type 1 & 1:1.13:1.7 & 28 & 42.44 & 4.85 & 5.12 & 2.35 \\
\hline 272 & 62 & $(60-180)$ & 0.34 & 0.4 & Type 1 & 1:1.13:1.7 & 60 & 47.69 & 4.94 & 5.18 & 2.36 \\
\hline 273 & 10 & $(0-10)$ & 0.34 & 0.5 & Type 2 & $1: 2.27: 4.22$ & 7 & 39.29 & 4.95 & 5.16 & 2.42 \\
\hline 274 & 10 & $(0-10)$ & 0.34 & 0.5 & Type 2 & $1: 2.27: 4.22$ & 14 & 40.18 & 4.99 & 5.26 & 2.46 \\
\hline 275 & 10 & $(0-10)$ & 0.34 & 0.5 & Type 2 & $1: 2.27: 4.22$ & 21 & 44.64 & 5.02 & 5.31 & 2.46 \\
\hline 276 & 10 & $(0-10)$ & 0.34 & 0.5 & Type 2 & $1: 2.27: 4.22$ & 28 & 41.07 & 5.06 & 5.35 & 2.47 \\
\hline 277 & 10 & $(0-10)$ & 0.34 & 0.5 & Type 2 & $1: 2.27: 4.22$ & 60 & $\mathbf{5 7 . 5 9}$ & 5.06 & 5.39 & 2.46 \\
\hline 278 & 10 & $(0-10)$ & 0.34 & 0.5 & Type 2 & $1: 2.27: 4.22$ & 90 & 49.55 & 5.10 & 5.42 & 2.46 \\
\hline 279 & 10 & $(0-10)$ & 0.34 & 0.5 & Type 2 & $1: 2.27: 4.22$ & 120 & 62.05 & 5.10 & 5.39 & 2.45 \\
\hline 280 & 10 & $(0-10)$ & 0.34 & 0.5 & Type 2 & $1: 2.27: 4.22$ & 150 & 42.41 & 5.11 & 5.42 & 2.47 \\
\hline 281 & 22 & $(10-30)$ & 0.34 & 0.4 & Type 3 & $1: 1.26: 2.45$ & 7 & 38.89 & 4.37 & 4.94 & 2.44 \\
\hline 282 & 22 & $(10-30)$ & 0.34 & 0.4 & Type 3 & 1:1.26:2.45 & 14 & 43.75 & 4.75 & 4.97 & 2.41 \\
\hline 283 & 22 & $(10-30)$ & 0.34 & 0.4 & Type 3 & $1: 1.26: 2.45$ & 21 & 46.41 & 4.79 & 5.00 & 2.43 \\
\hline 284 & 22 & $(10-30)$ & 0.34 & 0.4 & Type 3 & $1: 1.26: 2.45$ & 28 & 47.73 & 4.83 & 5.06 & 2.44 \\
\hline 285 & 22 & $(10-30)$ & 0.34 & 0.4 & Type 3 & $1: 1.26: 2.45$ & 60 & 45.96 & 4.89 & 5.09 & 2.43 \\
\hline 286 & 22 & $(10-30)$ & 0.34 & 0.4 & Type 3 & 1:1.26:2.46 & 120 & 49.50 & 4.81 & 5.07 & 2.43 \\
\hline 287 & 5 & $(0-10)$ & 2.05 & 0.6 & Type 2 & $1: 2.49: 6.72$ & 7 & 11.16 & 4.26 & 3.36 & 2.42 \\
\hline 288 & 5 & $(0-10)$ & 2.05 & 0.6 & Type 2 & $1: 2.49: 6.72$ & 16 & 15.63 & 4.58 & 4.03 & 2.42 \\
\hline 289 & 5 & $(0-10)$ & 2.05 & 0.6 & Type 2 & $1: 2.49: 6.72$ & 21 & 17.86 & 4.54 & 4.51 & 2.39 \\
\hline 290 & 5 & $(0-10)$ & 2.05 & 0.6 & Type 2 & $1: 2.49: 6.72$ & 28 & 14.73 & 4.60 & 4.68 & 2.41 \\
\hline 291 & 5 & $(0-10)$ & 2.05 & 0.6 & Type 2 & $1: 2.49: 6.72$ & 60 & 18.75 & 4.65 & 4.80 & 2.43 \\
\hline 292 & 5 & $(0-10)$ & 2.05 & 0.6 & Type 2 & $1: 2.49: 6.72$ & 90 & 24.78 & 4.69 & 4.79 & 2.39 \\
\hline 293 & 5 & $(0-10)$ & 2.05 & 0.6 & Type 2 & $1: 2.49: 6.72$ & 120 & 19.64 & 4.70 & 4.81 & 2.40 \\
\hline 294 & 15 & $(10-30)$ & 4.45 & 0.6 & Type 2 & $1: 2.02: 4.71$ & 7 & 17.86 & 4.47 & 4.53 & 2.34 \\
\hline 295 & 15 & $(10-30)$ & 4.45 & 0.6 & Type 2 & $1: 2.02: 4.71$ & 14 & 20.09 & 4.57 & 4.63 & 2.35 \\
\hline 296 & 15 & $(10-30)$ & 4.45 & 0.6 & Type 2 & $1: 2.02: 4.71$ & 21 & 21.43 & 4.59 & 4.65 & 2.35 \\
\hline 297 & 15 & $(10-30)$ & 4.45 & 0.6 & Type 2 & $1: 2.02: 4.71$ & 28 & 18.08 & 4.64 & 4.73 & 2.37 \\
\hline 298 & 15 & $(10-30)$ & 4.45 & 0.6 & Type 2 & $1: 2.02: 4.71$ & 60 & 30.80 & 4.70 & 4.80 & 2.37 \\
\hline 299 & 15 & $(10-30)$ & 4.45 & 0.6 & Type 2 & $1: 2.02: 4.71$ & 90 & 37.05 & 4.73 & 4.85 & 2.33 \\
\hline 300 & 15 & $(10-30)$ & 4.45 & 0.6 & Type 2 & $1: 2.02: 4.71$ & 120 & 24.55 & 4.75 & 4.84 & 2.35 \\
\hline 301 & 40 & $(30-60)$ & 4.45 & 0.6 & Type 2 & 1:1.91:3.88 & 7 & 12.50 & 4.19 & 3.94 & 2.40 \\
\hline 302 & 40 & $(30-60)$ & 4.45 & 0.6 & Type 2 & 1:1.91:3.88 & 14 & 16.52 & 4.62 & 4.06 & 2.36 \\
\hline 303 & 40 & $(30-60)$ & 4.45 & 0.6 & Type 2 & 1:1.91:3.88 & 21 & 17.86 & 4.36 & 4.45 & 2.35 \\
\hline 304 & 40 & $(30-60)$ & 4.45 & 0.6 & Type 2 & 1:1.91:3.88 & 28 & 15.63 & 4.41 & 4.58 & 2.37 \\
\hline 305 & 40 & $(30-60)$ & 4.45 & 0.6 & Type 2 & 1:1.91:3.88 & 60 & 22.32 & 4.45 & 4.64 & 2.37 \\
\hline 306 & 40 & $(30-60)$ & 4.45 & 0.6 & Type 2 & $1: 1.91: 3.88$ & 90 & 26.34 & 4.51 & 4.65 & 2.35 \\
\hline 307 & 40 & $(30-60)$ & 4.45 & 0.6 & Type 2 & 1:1.91:3.88 & 120 & 23.21 & 4.51 & 4.67 & 2.33 \\
\hline 308 & 70 & $(60-180)$ & 4.45 & 0.6 & Type 2 & $1: 1.87: 3.33$ & 7 & 14.73 & 4.17 & 3.95 & 2.34 \\
\hline
\end{tabular}


Table 1. Continued.

\begin{tabular}{|c|c|c|c|c|c|c|c|c|c|c|c|}
\hline $\begin{array}{c}\text { Sample } \\
\text { no. }\end{array}$ & $\begin{array}{c}\text { SLUMP } \\
(\mathbf{m m})\end{array}$ & $\begin{array}{c}\text { SLUMP } \\
\text { range } \\
(\mathbf{m m})\end{array}$ & $\begin{array}{c}\mathrm{SO}_{3} \% \text { in } \\
\text { fine } \\
\text { agregate }\end{array}$ & $\mathbf{W} / \mathbf{C}$ & $\begin{array}{l}\text { Coarse } \\
\text { Aggregate }\end{array}$ & $\begin{array}{c}\text { Mix } \\
\text { proportions }\end{array}$ & $\begin{array}{l}\text { Age } \\
\text { (day) }\end{array}$ & $\begin{array}{l}\text { Comp. } \\
\text { str. } \\
\text { (Mpa) }\end{array}$ & $\begin{array}{c}\text { Ult. } \\
\text { V(km/s) } \\
\text { direct }\end{array}$ & $\begin{array}{c}\text { Ult. } \\
\mathbf{V}(\mathbf{k m} / \mathbf{s}) \mathbf{s u} \\
\text { rface }\end{array}$ & $\begin{array}{l}\text { Density } \\
(\mathrm{gm} / \mathrm{cm} 3)\end{array}$ \\
\hline 309 & 70 & $(60-180)$ & 4.45 & 0.6 & Type 2 & $1: 1.87: 3.33$ & 14 & 19.20 & 4.25 & 4.38 & 2.35 \\
\hline 310 & 70 & $(60-180)$ & 4.45 & 0.6 & Type 2 & $1: 1.87: 3.33$ & 21 & 20.09 & 4.32 & 4.51 & 2.37 \\
\hline 311 & 70 & $(60-180)$ & 4.45 & 0.6 & Type 2 & $1: 1.87: 3.33$ & 28 & 20.09 & 4.38 & 4.54 & 2.37 \\
\hline 312 & 70 & $(60-180)$ & 4.45 & 0.6 & Type 2 & $1: 1.87: 3.33$ & 60 & 22.10 & 4.43 & 4.64 & 2.37 \\
\hline 313 & 70 & $(60-180)$ & 4.45 & 0.6 & Type 2 & 1:1.87:3.33 & 90 & 28.13 & 4.44 & 4.64 & 2.47 \\
\hline 314 & 70 & $(60-180)$ & 4.45 & 0.6 & Type 2 & $1: 1.87: 3.33$ & 120 & 26.34 & 4.47 & 4.64 & 2.33 \\
\hline 315 & 45 & $(30-60)$ & 0.34 & 0.6 & Type 1 & $1: 2.05: 3.21$ & 120 & 22.61 & 4.51 & 4.53 & 2.33 \\
\hline 316 & 45 & $(30-60)$ & 0.34 & 0.6 & Type 1 & 1:2.05:3.21 & 9 & 9.08 & 4.19 & 3.82 & 2.40 \\
\hline 317 & 45 & $(30-60)$ & 0.34 & 0.6 & Type 1 & $1: 2.05: 3.21$ & 14 & 10.59 & 4.62 & 3.94 & 2.36 \\
\hline 318 & 45 & $(30-60)$ & 0.34 & 0.6 & Type 1 & $1: 2.05: 3.21$ & 21 & 17.24 & 4.36 & 4.32 & 2.35 \\
\hline 319 & 45 & $(30-60)$ & 0.34 & 0.6 & Type 1 & $1: 2.05: 3.21$ & 28 & 20.12 & 4.41 & 4.44 & 2.37 \\
\hline 320 & 45 & $(30-60)$ & 0.34 & 0.6 & Type 1 & 1:2.05:3.21 & 60 & 21.61 & 4.45 & 4.49 & 2.37 \\
\hline 321 & 45 & $(30-60)$ & 0.34 & 0.6 & Type 1 & $1: 2.05: 3.21$ & 90 & 22.06 & 4.51 & 4.51 & 2.35 \\
\hline 322 & 50 & $(30-60)$ & 0.34 & 0.65 & Type 2 & $1: 2.31: 3.47$ & 14 & 18.30 & 4.39 & 4.62 & 2.33 \\
\hline 323 & 50 & $(30-60)$ & 0.34 & 0.65 & Type 2 & $1: 2.31: 3.47$ & 21 & 20.98 & 4.22 & 4.66 & 2.33 \\
\hline 324 & 50 & $(30-60)$ & 0.34 & 0.65 & Type 2 & $1: 2.31: 3.47$ & 28 & 23.66 & 4.48 & 4.70 & 2.33 \\
\hline 325 & 50 & $(30-60)$ & 0.34 & 0.65 & Type 2 & 1:2.31:3.47 & 60 & 28.57 & 4.55 & 4.74 & 2.32 \\
\hline 326 & 50 & $(30-60)$ & 0.34 & 0.65 & Type 2 & 1:2.31:3.47 & 90 & 27.68 & 4.58 & 4.77 & 2.33 \\
\hline 327 & 20 & $(10-30)$ & 0.34 & 0.8 & Type 2 & 1:3.37:5.06 & 14 & 9.15 & 4.18 & 4.15 & 2.40 \\
\hline 328 & 20 & $(10-30)$ & 0.34 & 0.8 & Type 2 & $1: 3.37: 5.06$ & 21 & 12.95 & 4.39 & 4.46 & 2.38 \\
\hline 329 & 20 & $(10-30)$ & 0.34 & 0.8 & Type 2 & $1: 3.37: 5.06$ & 28 & 16.16 & 4.40 & 4.47 & 2.37 \\
\hline 330 & 20 & $(10-30)$ & 0.34 & 0.8 & Type 2 & $1: 3.37: 5.06$ & 60 & 19.87 & 4.46 & 4.51 & 2.34 \\
\hline 331 & 20 & $(10-30)$ & 0.34 & 0.8 & Type 2 & $1: 3.37: 5.06$ & 90 & 16.74 & 4.43 & 4.49 & 2.34 \\
\hline 332 & 28 & $(10-30)$ & 0.34 & 0.45 & Type 3 & 1:1.51:2.79 & 7 & 26.08 & 4.46 & 4.61 & 2.41 \\
\hline 333 & 28 & $(10-30)$ & 0.34 & 0.45 & Type 3 & 1:1.51:2.79 & 14 & $\mathbf{3 7 . 1 3}$ & 4.64 & 4.88 & 2.42 \\
\hline 334 & 28 & $(10-30)$ & 0.34 & 0.45 & Type 3 & 1:1.51:2.79 & 21 & 38.01 & 4.70 & 4.93 & 2.41 \\
\hline 335 & 28 & $(10-30)$ & 0.34 & 0.45 & Type 3 & 1:1.51:2.79 & 28 & 42.43 & 4.71 & 5.00 & 2.41 \\
\hline 336 & 28 & $(10-30)$ & 0.34 & 0.45 & Type 3 & 1:1.51:2.79 & 60 & 44.20 & 4.78 & 5.08 & 2.42 \\
\hline 337 & 28 & $(10-30)$ & 0.34 & 0.45 & Type 3 & 1:1.51:2.79 & 90 & 50.83 & 4.81 & 5.08 & 2.41 \\
\hline 338 & 100 & $(60-180)$ & 0.34 & 0.8 & Type 1 & $1: 3.33: 3.33$ & 7 & 6.70 & 3.66 & 3.00 & 2.34 \\
\hline 339 & 100 & $(60-180)$ & 0.34 & 0.8 & Type 1 & $1: 3.33: 3.33$ & 21 & 16.96 & 4.28 & 4.40 & 2.38 \\
\hline 340 & 100 & $(60-180)$ & 0.34 & 0.8 & Type 1 & $1: 3.33: 3.33$ & 28 & 17.41 & 4.28 & 4.44 & 2.37 \\
\hline 341 & 100 & $(60-180)$ & 0.34 & 0.8 & Type 1 & $1: 3.33: 3.33$ & 60 & 20.98 & 4.36 & 4.52 & 2.36 \\
\hline 342 & 100 & $(60-180)$ & 0.34 & 0.8 & Type 1 & $1: 3.33: 3.33$ & 90 & 20.09 & 4.40 & 4.52 & 2.37 \\
\hline 343 & 72 & $(60-180)$ & 0.34 & 0.45 & Type 1 & 1:1.47:1.86 & 7 & 33.69 & 4.38 & 4.58 & 2.37 \\
\hline 344 & 72 & $(60-180)$ & 0.34 & 0.45 & Type 1 & 1:1.47:1.86 & 14 & 32.81 & 4.49 & 4.69 & 2.37 \\
\hline 345 & 72 & $(60-180)$ & 0.34 & 0.45 & Type 1 & 1:1.47:1.86 & 21 & 33.25 & 4.53 & 4.79 & 2.38 \\
\hline 346 & 72 & $(60-180)$ & 0.34 & 0.45 & Type 1 & 1:1.47:1.86 & 28 & 38.50 & 4.55 & 4.83 & 2.37 \\
\hline 347 & 72 & $(60-180)$ & 0.34 & 0.45 & Type 1 & 1:1.47:1.86 & 60 & 43.75 & 4.62 & 4.92 & 2.36 \\
\hline 348 & 72 & $(60-180)$ & 0.34 & 0.45 & Type 1 & 1:1.47:1.86 & 90 & 45.50 & 4.67 & 4.89 & 2.36 \\
\hline 349 & 72 & $(60-180)$ & 0.34 & 0.45 & Type 1 & 1:1.47:1.86 & 120 & 45.50 & 4.66 & 4.89 & 2.36 \\
\hline 350 & 28 & $(10-30)$ & 0.34 & 0.6 & Type 3 & $1: 2.25: 4.6$ & 120 & 27.79 & 4.75 & 4.68 & 2.35 \\
\hline 351 & 28 & $(10-30)$ & 0.34 & 0.6 & Type 3 & $1: 2.25: 4.6$ & 7 & 18.96 & 4.47 & 4.39 & 2.34 \\
\hline 352 & 28 & $(10-30)$ & 0.34 & 0.6 & Type 3 & $1: 2.25: 4.6$ & 14 & 21.49 & 4.57 & 4.49 & 2.35 \\
\hline 353 & 28 & $(10-30)$ & 0.34 & 0.6 & Type 3 & $1: 2.25: 4.6$ & 21 & 22.07 & 4.59 & 4.51 & 2.35 \\
\hline 354 & 28 & $(10-30)$ & 0.34 & 0.6 & Type 3 & $1: 2.25: 4.6$ & 28 & 24.42 & 4.64 & 4.59 & 2.37 \\
\hline 355 & 28 & $(10-30)$ & 0.34 & 0.6 & Type 3 & $1: 2.25: 4.6$ & 60 & 26.59 & 4.70 & 4.65 & 2.37 \\
\hline 356 & 28 & $(10-30)$ & 0.34 & 0.6 & Type 3 & $1: 2.25: 4.6$ & 90 & 28.30 & 4.73 & 4.70 & 2.33 \\
\hline 357 & 95 & $(60-180)$ & 0.34 & 0.8 & Type 4 & 1:3.35:4.27 & 7 & 6.70 & 4.10 & 3.18 & 2.39 \\
\hline 358 & 95 & $(60-180)$ & 0.34 & 0.8 & Type 4 & $1: 3.35: 4.27$ & 14 & 13.39 & 4.45 & 4.40 & 2.39 \\
\hline 359 & 95 & $(60-180)$ & 0.34 & 0.8 & Type 4 & 1:3.35:4.27 & 28 & 16.74 & 4.52 & 4.54 & 2.37 \\
\hline 360 & 95 & $(60-180)$ & 0.34 & 0.8 & Type 4 & $1: 3.35: 4.27$ & 60 & 21.88 & 4.58 & 4.58 & 2.37 \\
\hline 361 & 95 & $(60-180)$ & 0.34 & 0.8 & Type 4 & 1:3.35:4.27 & 90 & 20.98 & 4.60 & 4.61 & 2.37 \\
\hline 362 & 55 & $(30-60)$ & 0.34 & 0.8 & Type 4 & $1: 3.2: 5.23$ & 10 & 13.84 & 4.45 & 4.00 & 2.42 \\
\hline 363 & 55 & $(30-60)$ & 0.34 & 0.8 & Type 4 & $1: 3.2: 5.23$ & 21 & 17.19 & 4.58 & 4.57 & 2.41 \\
\hline 364 & 55 & $(30-60)$ & 0.34 & 0.8 & Type 4 & $1: 3.2: 5.23$ & 28 & 22.77 & 4.65 & 4.56 & 2.41 \\
\hline 365 & 55 & $(30-60)$ & 0.34 & 0.8 & Type 4 & $1: 3.2: 5.23$ & 60 & 24.33 & 4.66 & 4.63 & 2.40 \\
\hline 366 & 55 & $(30-60)$ & 0.34 & 0.8 & Type 4 & $1: 3.2: 5.23$ & 90 & 20.54 & 4.67 & 4.58 & 2.40 \\
\hline 367 & 90 & $(60-180)$ & 0.34 & 0.8 & Type 1 & $1: 3.35: 4.27$ & 7 & 6.76 & 4.15 & 3.21 & 2.39 \\
\hline 368 & 90 & $(60-180)$ & 0.34 & 0.8 & Type 1 & 1:3.35:4.27 & 90 & 21.19 & 4.64 & 4.65 & 2.37 \\
\hline 369 & 90 & $(60-180)$ & 0.34 & 0.8 & Type 1 & 1:3.35:4.27 & 14 & 13.53 & 4.50 & 4.44 & 2.39 \\
\hline 370 & 90 & $(60-180)$ & 0.34 & 0.8 & Type 1 & 1:3.35:4.27 & 28 & 16.91 & 4.57 & 4.58 & 2.37 \\
\hline 371 & 90 & $(60-180)$ & 0.34 & 0.8 & Type 1 & $1: 3.35: 4.27$ & 60 & 22.09 & 4.63 & 4.63 & 2.37 \\
\hline 372 & 10 & $(0-10)$ & 0.34 & 0.9 & Type 2 & $1: 5.29: 6.68$ & 14 & 8.93 & 4.13 & 4.19 & 2.33 \\
\hline 373 & 10 & $(0-10)$ & 0.34 & 0.9 & Type 2 & $1: 5.29: 6.68$ & 21 & 10.71 & 4.35 & 4.45 & 2.38 \\
\hline 374 & 10 & $(0-10)$ & 0.34 & 0.9 & Type 2 & $1: 5.29: 6.68$ & 28 & 12.50 & 4.35 & 4.50 & 2.36 \\
\hline 375 & 10 & $(0-10)$ & 0.34 & 0.9 & Type 2 & $1: 5.29: 6.68$ & 60 & 16.29 & 4.47 & 4.53 & 2.37 \\
\hline 376 & 10 & $(0-10)$ & 0.34 & 0.9 & Type 2 & $1: 5.29: 6.68$ & 90 & 15.63 & 4.51 & 4.52 & 2.34 \\
\hline 377 & 58 & $(30-60)$ & 0.34 & 0.45 & Type 2 & 1:1.4:2.24 & 120 & 44.64 & 4.81 & 4.89 & 2.35 \\
\hline 378 & 58 & $(30-60)$ & 0.34 & 0.45 & Type 2 & 1:1.4:2.24 & 7 & 29.61 & 4.48 & 4.50 & 2.34 \\
\hline 379 & 58 & $(30-60)$ & 0.34 & 0.45 & Type 2 & 1:1.4:2.24 & 14 & 30.27 & 4.62 & 4.68 & 2.34 \\
\hline 380 & 58 & $(30-60)$ & 0.34 & 0.45 & Type 2 & 1:1.4:2.24 & 21 & 34.47 & 4.65 & 4.77 & 2.33 \\
\hline 381 & 58 & $(30-60)$ & 0.34 & 0.45 & Type 2 & $1: 1.4: 2.24$ & 28 & 36.24 & 4.67 & 4.83 & 2.35 \\
\hline 382 & 58 & $(30-60)$ & 0.34 & 0.45 & Type 2 & $1: 1.4: 2.24$ & 60 & 46.41 & 4.75 & 4.92 & 2.33 \\
\hline 383 & 58 & $(30-60)$ & 0.34 & 0.45 & Type 2 & 1:1.4:2.24 & 90 & 45.08 & 4.79 & 4.92 & 2.35 \\
\hline
\end{tabular}


Table 2. Grading and characteristics of coarse aggregate used.

\begin{tabular}{|c|c|c|c|c|c|}
\hline \multirow{2}{*}{$\begin{array}{c}\text { Sieve Size } \\
\text { mm }\end{array}$} & \multicolumn{5}{|c|}{ Passing Percentage \% } \\
\cline { 2 - 6 } & Type 1 & Type 2 & Type 3 & Type 4 & Type 5 \\
\hline 37.5 & 100 & 100 & 100 & 100 & 100 \\
\hline 20 & 70 & 100 & 100 & 100 & 100 \\
\hline 14 & 40 & 70 & 100 & 100 & 100 \\
\hline 10 & 10 & 40 & 50 & 100 & 0 \\
\hline 5 & 0 & 0 & 0 & 0 & 0 \\
\hline
\end{tabular}

Table 3. Comparison between SUPV and DUPV

\begin{tabular}{|c|c|c|}
\hline UPV Type & Correlation Factor & $\mathbf{R}^{\mathbf{2}}$ Value \\
\hline SUPV & 0.8329 & 0.7055 \\
\hline DUPV & 0.7389 & 0.6504 \\
\hline
\end{tabular}

Figures 1 and 2 show that the rate variations of the DUPV are less than the variations of SUPV for the same variation of the compressive strength. Thus, an increase in the DUPV happens at a lesser rate than increasing the compressive strength. The SUPV seems to be more sensitive to the increase in compressive strength. This tendency is noticed since the propagation of surface waves is restricted to a region near the boundaries of the free external surface of the material. The depth of the penetration is on the order of one wavelength thickness. The cement paste content of this layer is greater than the average paste content inside the concrete due to the so-called wall effect. Therefore, the velocity of a surface wave SUPV is influenced more by the paste properties than that of the direct waves DUPV that travel through the whole mass of the concrete. Since the concrete strength is also controlled by the strength of the hardened cement paste, SUPV may be a better indicator of the concrete strength than DUPV.

Figure 3 shows that for pulse velocity less than $(4.5 \mathrm{~km} / \mathrm{s})$, the (DUPV) was more significant than the (SUPV) for the same compressive strength. For pulse velocity greater than $(4.5 \mathrm{~km} / \mathrm{s})$, the (DUPV) was less than the (SUPV) for the same compressive strength. This happens because, at low pulse velocity (less than $4.5 \mathrm{~km} / \mathrm{s}$ ), the ultrasonic wave passing throws the coarse aggregate and gives us high (DUPV) when the compressive strength low. The (SUPV) wave passing throw the cement mortar and will represent the compressive strength more accurately. Besides that, the correlation factor of SUPV was more significant than (DUPV), so using (SUPV) is better than using the (DUPV) to represent the relationship with the compressive strength.

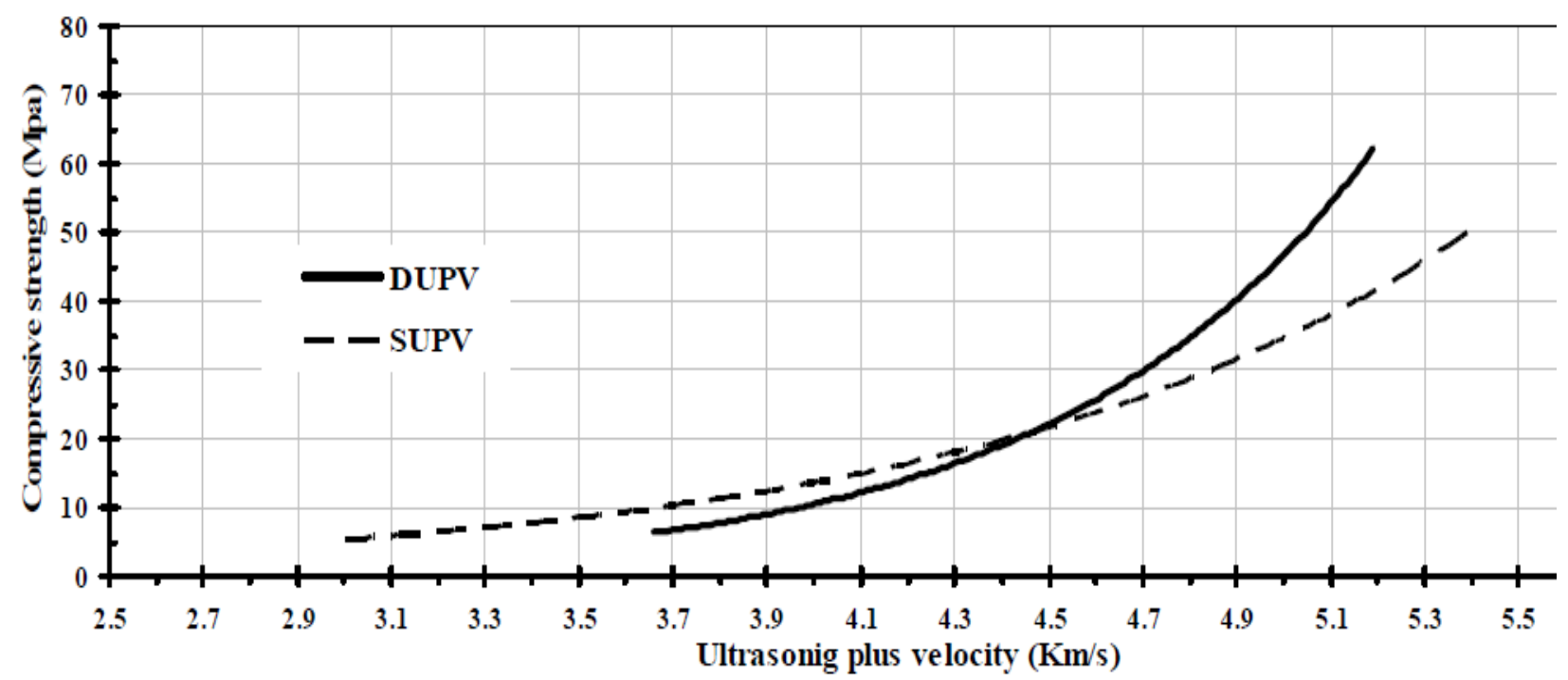

Figure 3. Relation between (DUPV and SUPV) with the compressive strength for concrete samples subjected to normal curing. 


\section{Results of Statistical Analysis}

The statistical methods can explain the test results and the prediction of concrete strength, in the case of the test that was carried out in a satisfactory way and standard tools. The statistical methods were proved to have good values. The goal of a regression method is to fit a line through points (results) so that the squared deviations of the observed points from that line are minimized. In statistical modeling, the overall objective is to develop a predictive equation relating a criterion variable to one or more predictor variables. In this research, the criterion variables include the compressive strength, the direct ultrasonic wave, and the surface (indirect) waves. The summary of descriptive statistics of all the variables is shown in Table 4, and the correlation matrix for the data are shown in Table 5. The terms shown in Table 4 and 5 are defined as follows: $\mathrm{C}=$ Compressive strength $(\mathrm{MPa}), \mathrm{D}=$ Direct ultrasonic wave velocity $(\mathrm{km} / \mathrm{s}), \mathrm{S}=$ Surface (indirect) ultrasonic wave velocity $(\mathrm{km} / \mathrm{s})$

Table 4. Statistical summary for predictor and criteria variables.

\begin{tabular}{|c|c|c|c|c|c|c|c|c|}
\hline Variable & $\mathbf{N}$ & Range & Min. & Max. & Sum & Mean & $\begin{array}{c}\text { Mean. } \\
\text { Std. Error }\end{array}$ & $\begin{array}{c}\text { Std. } \\
\text { dev. }\end{array}$ \\
\hline C & 383 & 59.47 & 5.26 & 64.73 & 12248.1 & 31.979 & 0.6299 & 12.3273 \\
\hline D & 381 & 1.53 & 3.66 & 5.19 & 1774.02 & 4.6562 & 0.0120 & 0.2353 \\
\hline S & 383 & 2.42 & 3 & 5.42 & 1830.51 & 4.7794 & 0.2032 & 0.3976 \\
\hline
\end{tabular}

Table 5. Correlation matrix for predictor and criteria variables.

\begin{tabular}{|c|c|c|c|}
\hline Variable & C & D & S \\
\hline C & 1 & 0.760 & 0.810 \\
\hline $\mathbf{D}$ & 0.760 & 1 & 0.880 \\
\hline S & 0.810 & 0.880 & 1 \\
\hline
\end{tabular}

Compressive Strength Modeling. The relation between compressive strength and the ultrasonic velocity (DandS) is exponential and found from the previous studies $[1,3,5]$ and others. Therefore, to catch these predictor variables in one equation, it's proposed to take the exponential value of the (UPV) data (DandS reading) and choose the linear regression to specify the parameter and select the stepwise.

$$
\mathrm{C}=0.128 \mathrm{e}^{1.127 \mathrm{~S}}
$$

The correlation of the proposed equation is $(0.84726)$, and $\mathrm{R}^{2}$ is $(0.7883)$. Verification of the proposed equation and previous equations depends on separated data taken from a different source. The data adopted for the study in this part was taken from ref erences[9,10], as shown in Table 6 .

Table 6. Comprising data from Neville $[9,10]$.

\begin{tabular}{|c|c|c|c|c|c|c|c|c|c|}
\hline Compressive Strength (MPa) & 17 & 20.5 & 21 & 28 & 31.5 & 31 & 42 & 51 & 52.5 \\
\hline Ultrasonic Velocity (km/s) & 3.75 & 3.9 & 4.1 & 4.3 & 4.3 & 4.4 & 4.4 & 4.6 & 4.7 \\
\hline
\end{tabular}

By using the direct ultrasonic velocity illustrated in Table 6, the compressive strength estimated from some of the previous equations and also estimated from the proposed Eq. (7), which depending on the SUPV. The equivalent surface SUPV was taken from Eq. (8), built only for verification purposes from the research data.

$\mathrm{S}=1.405 \mathrm{D}-1.406$

where: $\mathrm{S}$ is a surface ultrasonic velocity and $\mathrm{D}$ is a direct ultrasonic velocity in $\mathrm{km} / \mathrm{sec}$

From Figure 4 one can notice that no equation can satisfy all the points entirely. The proposed equation could be considered the nearest one to the points taken from reference [9], and that agrees with the correlation factor appear in Table 7, which equals (0.9611). 
Table 7. Correlation factor for proposed and previous equations.

\begin{tabular}{|c|c|c|c|c|c|c|}
\hline Equation & $\begin{array}{c}\text { Elvery and Ibrahim } \\
{[\mathbf{2}]}\end{array}$ & $\begin{array}{c}\text { Raouf and } \\
\text { Ali [3] }\end{array}$ & $\begin{array}{c}\text { Jones } \\
{[\mathbf{1}]}\end{array}$ & $\begin{array}{c}\text { Nash't et al. } \\
{[\mathbf{5}]}\end{array}$ & $\begin{array}{c}\text { Pundit } \\
\text { Manual }\end{array}$ & Proposed \\
\hline Cor. Coef. & 0.9591 & 0.9469 & 0.9447 & 0.9495 & 0.9565 & 0.9611 \\
\hline
\end{tabular}

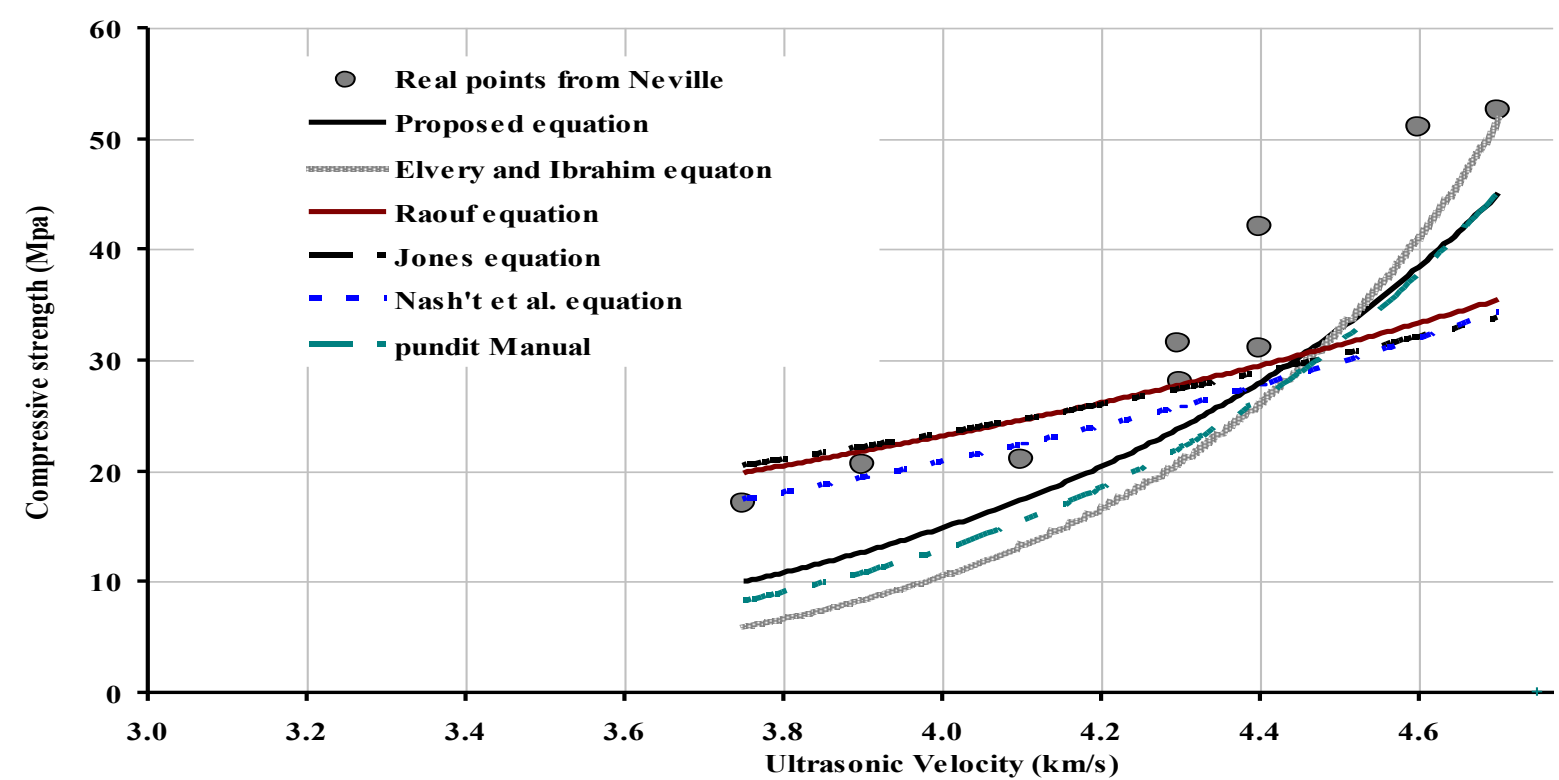

Figure 4. Relation between compressive strength and ultrasonic pulse velocity for proposed and previous equations.

Effect of The Salts on The Proposed Relation. The salt content in fine aggregate affects the relationship between the compressive strength and the (SUPV). Suppose the inspector suspects that there's salt in the tested concrete and to be on the safe side, the following correction of the (SUPV) reading should be used before using Eq. (7). This correction was found from separating the normal curing data for concrete with salt from the data of concrete with no salt. These two groups of data are interred in the SPSS program, and with two equations are found one for the case of high salt content, and the other represents the data for concrete with a bit of salt, as mentioned earlier

$$
\mathrm{C}=0.8983 \mathrm{e}^{0.706 \mathrm{~s}^{\prime}}
$$

By equating Eq. (7) with Eq. (8), the relation between (SUPV) for concrete with high salt and concrete with a little salt is found as shown in Eq. (10).

$$
S=0.63 S^{\prime}+1.72
$$

where:

$S=$ Surface (indirect) ultrasonic wave velocity ( $\mathrm{km} / \mathrm{s})$ (with no salt)

$\mathrm{S}^{\prime}=$ Surface ultrasonic wave velocity $(\mathrm{km} / \mathrm{s})$ (with salt)

\section{Conclusions}

- The SUPV is more sensitive than the DUPV for assessment of compressive strength where $\mathrm{R}^{2}$ value equal to $(0.7883)$ and correlation factor equal to $(0.84726)$ for equation depending on surface reading while $R^{2}$ value equal to $(0.6504)$ and correlation factor equal to $(0.7389)$ for equation depend on direct reading.

- $\mathrm{SO}_{3}$ content in fine aggregate effect decreases compression strength while the SUPV reading does not affect the same rate. For using the general proposed equation, the proposed correction of SUPV reading must be used. 
- For the same sample, when concrete compressive strength is more than (20.5 MPa), the SUPV reading is more significant than DUPV, while for compressive strength less than $(20.5 \mathrm{MPa})$, the DUPV is greater than SUPV.

\section{References}

[1] Jones, R., 1962. Non destructive testing of concrete. London, Cambridge University.

[2] Elvery, R.H., and Ibrahim,L.A.M., 1976. Ultasonic assessment of concrete strength at early ages. Magazine of Concrete Research, 28(97), pp.181-190.

[3] Raouf, Z. and Ali, Z. M., 1983. Assessment of concrete characteristics at an early age by ultrasonic pulse velocity. Journal of Building Research, 2(1), pp.31-44.

[4] Popovics, S., Joseph, L. R., John, S. P., 1990. The behavior of ultrasonic pulses in concrete. Cement and Concrete Research, 20(2), pp.259-270.

[5] Nash't, I. H., A'bour S.H., Sadoon A.A., 2005. Finding an unified relationship between crushing strength of concrete and non-destructive tests. 3rd MENDT - Middle East Nondestructive Testing Conference and Exhibition, Bahrain, Manama.

[6] Jassim, A. K., 2012. Prediction of compressive strength of reinforced concrete structural members by using combined non-destructive tests. M.Sc. Thesis, University of Baghdad, Iraq.

[7] Fawzi, N. M., Said, A. I., and Jassim, A. K., 2013. Prediction of compressive strength of reinforced concrete structural elements by using combined non-destructive tests. Journal of Engineering, 19(10), pp. 1189-1121.

[8] Ali B. A. H, 2008. Assessment of Concrete compressive strength by ultrasonic non-destructive test. M.Sc. Thesis, University of Baghdad, Iraq.

[9] Nivelle, A. M., 1995. Properties of Concrete. 4th Edition Longman Group Limited.

[10] Sturrup V.R. , Vecchio F.J. and Caratin H, 1984. Pulse velocity as a measure of concrete compressive strength, in situ/non-destructive testing of concrete. Ed.V.M. Malhotra, ACI SP-82, Detroit Michigan, pp. 201-227. 